\title{
Geologische, hydrogeologische und anthropogene Einflüsse auf die Wasserchemie der Möhlin, Schwarzwald - eine Momentaufnahme
}

\author{
Niels Nitzsche ${ }^{1}$ (D) $\cdot$ Wolfgang Siebel $^{1} \cdot$ Ingrid Stober $^{1}$ \\ Eingegangen: 17. August 2020 / Überarbeitet: 18. November 2020 / Angenommen: 25. Januar 2021 / Online publiziert: 22. Februar 2021 \\ (c) Der/die Autor(en) 2021
}

\section{Zusammenfassung}

Diese Arbeit untersucht die geologischen, hydrogeologischen und anthropogenen Einflüsse auf die chemische Zusammensetzung eines Flusses, am Beispiel der Möhlin. Wasserproben wurden an verschiedenen Flussabschnitten genommen und mittels Alkalinitätstest und Ionenchromatographie auf die Ionen $\mathrm{Ca}^{2+}, \mathrm{Mg}^{2+}, \mathrm{Na}^{+}, \mathrm{K}^{+}, \mathrm{HCO}_{3}^{-}, \mathrm{CO}_{3}{ }^{2-}, \mathrm{SO}_{4}{ }^{2-}, \mathrm{Cl}^{-}, \mathrm{F}^{-}$und $\mathrm{NO}_{3}{ }^{-}$ untersucht. Daneben wurden Leitfähigkeit, Temperatur, Abfluss und $\mathrm{pH}-$ Wert gemessen und die $\delta \mathrm{D}-$ und $\delta^{18} \mathrm{O}-$-Isotopenzusammensetzung durch Cavity-Ring-Down-Spektroskopie ermittelt. Die Untersuchung zeigt, dass es sich bei der Möhlin im Oberlauf um einen silikatisch geprägten Fluss handelt, während sie sich im Unterlauf an der Grenze von silikatisch bis karbonatisch bewegt. Die Geologie hat den stärksten Einfluss auf die Wasserchemie der Möhlin, wobei große Anteile am geologischen Fingerabdruck durch ihre Zuflüsse geprägt werden. Weitere Einflussfaktoren sind anthropogene Einleitungen, wasserbauliche Maßnahmen sowie der ehemalige Bergbau im Elsass und Schwarzwald. Insgesamt weist das Wasser der Möhlin in Bezug auf die untersuchten Parameter eine gute Wasserqualität auf.

Schlüsselwörter Hydrogeologie $\cdot$ Wasserchemie $\cdot$ Wasserisotopie $\cdot$ Schwarzwald $\cdot$ Oberrheingraben

\section{A snapshot of the geological, hydrogeological and anthropogenic impacts on the water chemistry of the Möhlin River, Black Forest}

\begin{abstract}
The subject of this study is the examination of the chemical composition of the Möhlin River with respect to its geology, hydrogeology and potential anthropogenic impacts. Water samples taken at different locations in the river were analyzed for $\mathrm{Ca}^{2+}, \mathrm{Mg}^{2+}, \mathrm{Na}^{+}, \mathrm{K}^{+}, \mathrm{HCO}_{3}^{-}, \mathrm{CO}_{3}^{2-}, \mathrm{SO}_{4}^{2-}, \mathrm{Cl}^{-}, \mathrm{F}^{-}$and $\mathrm{NO}_{3}{ }^{-}$via alkalinity testing and ion chromatography. Electric conductivity, temperature, discharge and $\mathrm{pH}$-values were measured as well as stable isotopes $\left(\delta \mathrm{D}, \delta^{18} \mathrm{O}\right)$, which were analyzed by Cavity-Ring-Down-Spectroscopy. In the upper section, the Möhlin River can be classified as a siliceous water body, while the lower part of the river is at the transition between a siliceous and carbonatic water body. Out of all impact factors, the geology is the most important, while inflows also play a key role. Other impact factors on the water chemistry are anthropogenic discharges, hydraulic-engineering actions and legacy mining activities in the Alsace and the Black Forest regions. Despite these factors, the water quality of the Möhlin River can be classified as "good" in relation to the measured parameters.
\end{abstract}

Keywords Catchment geology · Water chemistry · Water isotopes · Black Forest · Upper Rhine valley

Niels Nitzsche
niels.nitzsche@gmx.de
Wolfgang Siebel
wolfgang.siebel@uni-tuebingen.de
Ingrid Stober
ingrid.stober@minpet.uni-freiburg.de
Institut für Geo- und Umweltnaturwissenschaften,
Albert-Ludwigs-Universität Freiburg im Breisgau,
Tennenbacher Str. 4, 79106 Freiburg, Deutschland 


\section{Einleitung}

Die Wasserrahmenrichtlinie der EU (Richtlinie 2000/60/ EG), welche sich zum Ziel setzt, bis 2027 unter anderem einen „chemisch guten“ Zustand in den Gewässern der EU-Mitgliedsstaaten zu erreichen, hebt die Wichtigkeit von Untersuchungen und Monitoring von Fließgewässern in Deutschland hervor. Um anthropogene Einträge und Verunreinigungen verlässlich zu bestimmen, müssen die unterschiedlichen Eintragspfade von Ionen in ein Fließgewässer genau charakterisiert werden. Durch eine Vielzahl von vorangegangenen Studien und die damit verbundene Entwicklung von Leitbildern zur Charakterisierung geologischer Hintergründe (wie z. B. LAWA 1998; Schneider et al. 2002; Koelle 2010) können die eingetragenen Hauptionen in der Regel verlässlich verschiedenen Quellen zugeordnet werden.

Die in einem Fließgewässer anwesenden Hauptionen geben Aufschluss über den geologischen Untergrund sowie über anthropogene Nutzungen und damit verbundene Verunreinigungen. Ionen werden durch Verwitterungsvorgänge aus den Mineralen und Gesteinen gelöst und gelangen auf natürliche Weise in das Gewässer. Die unterschiedlichen Zusammensetzungen der Gesteine sowie die Kinetik der Verwitterungsvorgänge ergeben somit einen ,geologischen Fingerabdruck" des Gewässers. Entsprechend unserem Kenntnisstand über Fließgewässer, können Ionenkonzentrationen auf ihre Eintragsquellen zurückgeführt und interpretiert werden.

Ziel dieser Untersuchung ist es, den Einfluss der Geologie, vor allem der Lithologie, sowie der Hydrologie auf die chemische Zusammensetzung der Hauptionen eines Fließgewässers, in diesem Fall die Möhlin südlich von Freiburg, qualitativ und quantitativ zu erfassen. Hauptteil der Arbeit sind die Konzentrationsmessungen der im Möhlinwasser vorhandenen Hauptionen (Calcium $\left(\mathrm{Ca}^{2+}\right)$, Magnesium $\left(\mathrm{Mg}^{2+}\right)$, Natrium $\left(\mathrm{Na}^{+}\right)$, Kalium $\left(\mathrm{K}^{+}\right)$, Hydrogenkarbonat $\left(\mathrm{HCO}_{3}^{-}\right)$, Karbonat $\left(\mathrm{CO}_{3}{ }^{2-}\right)$, Sulfat $\left(\mathrm{SO}_{4}{ }^{2-}\right)$, Chlorid $\left(\mathrm{Cl}^{-}\right)$ sowie Fluor $\left(\mathrm{F}^{-}\right)$und des Nährstoffes Nitrat $\left(\mathrm{NO}_{3}^{-}\right)$) sowie die anschließende Interpretation der Menge und Herkunft dieser Ionen. $\delta^{18} \mathrm{O}$ - und $\delta^{2} \mathrm{H}$-Isotopenzusammensetzungen wurden bestimmt, um Rückschlüsse über die Herkunft des Wassers zu erhalten und somit Einflüsse von anderen Oberflächengewässern auf die Möhlin erkennen zu können. Zur verbesserten Interpretation wurden auch die Leitparameter Temperatur, Leitfähigkeit, pH-Wert sowie an einigen Stellen die Höhe des Abflusses ermittelt. Die vorliegende Arbeit basiert teilweise auf einer Bachelorarbeit (Nitzsche 2019), welche weitergehend ausgewertet und interpretiert wurde.

Die Untersuchungen wurden während einer Niedrigwassersituation in der Möhlin durchgeführt. Diesem Vorgehen liegt die Tatsache zugrunde, dass geologische Hintergründe in der Wasserchemie bei geringem Abfluss wegen der fehlenden Verdünnung durch Niederschlag deutlich stärker zutage treten, als bei hohem Abfluss. Auch die verschiedenen Einflüsse der Zuflüsse in die Möhlin wurden im Rahmen dieser Untersuchung sichtbar gemacht. Die Möhlin wurde als Untersuchungsobjekt gewählt, da sie auf relativ kurzer Distanz mit Schwarzwald, Vorbergzone und Oberrheingraben völlig unterschiedliche geologische Einheiten und insgesamt eine Vielzahl verschiedener geologischer Formationen durchläuft und daher Aufschluss geben kann, wie schnell und wie signifikant sich geologische Änderungen in der Wasserchemie eines Fließgewässers widerspiegeln. Der mittlere und untere Flussabschnitt liegt in einem landwirtschaftlich genutzten Gebiet, in dem vor allem das Grundwasser Erhöhungen anthropogener Einträge erfährt (Albrecht 2006). Ein weiteres Augenmerk war darauf gerichtet, wie stark sich diese Belastung auf die Möhlin und ihre Zuflüsse auswirkt.

\section{Untersuchungsgebiet}

Die Möhlin durchfließt Teile des Schwarzwalds, der Vorbergzone und des Oberrheingrabens südlich von Freiburg im Breisgau und weist eine Länge von etwa $32 \mathrm{~km}$ auf (Abb. 1). Der Fluss entspringt südwestlich des Schauinslandgipfels im Hochschwarzwald auf einer Höhe von $1088 \mathrm{~m}$ über NN, fließt zunächst nach Westen und bahnt sich seinen Weg durch die Ortschaften St. Ulrich, Ehrenstetten und Hausen an der Möhlin, bevor er südlich von Breisach auf $188 \mathrm{~m}$ über NN in den Rhein mündet. Das Einzugsgebiet (EZG) der Möhlin hat eine Fläche von $228 \mathrm{~km}^{2}$ (Seidel und Mäckel 2007). Die Höhendifferenz der Möhlin beträgt etwa $900 \mathrm{~m}$. Das durchschnittliche Gefälle liegt bei $2,87 \%$. Etwa $90 \%$ der Höhenmeter werden in den ersten $10 \mathrm{~km}$ des Flussverlaufs überwunden, wobei Gefälle von $35-40 \%$ erreicht werden.

\section{Hydrologie und Hydrogeologie}

Der Jahresniederschlag des Untersuchungsgebiets liegt zwischen 800 und $1300 \mathrm{~mm}$, wobei die Höchstwerte im Quellgebiet am Schauinsland erreicht werden (DWD 2019) und geringere Niederschlagswerte im Oberrheingraben anzutreffen sind. Der mittlere Abfluss der Möhlin am Pegel Oberambringen (OA, Abb. 1) liegt bei 6601/s (Hochwasservorhersagezentrale Baden-Württemberg 2019). Die Möhlin ist im Oberlauf weitgehend naturbelassen mit vielen Stufen im heterogenen Flussbett. Im unteren Flussverlauf etwa ab Ehrenstetten wurde sie im 19. Jahrhundert komplett begradigt und mit einer künstlichen Böschung versehen (Seidel und Mäckel 2007). Dies führt, überschattet von der Rheinbegradigung und gemeinsam mit einer generellen Intensivierung der Landwirtschaft, zu einer Absenkung des Grund- 
Abb. 1 Geologische Einheiten im Einzugsgebiet der Möhlin, Probennahmepunkte, sowie der Flussverlauf von Möhlin, Ahbach, Eckbach, Neumagen und Seltenbach. Die Geologie basiert auf einer Karte von LUBW (2012). UM und OA repräsentiert die Pegel Untermünstertal (UM) und Oberambringen (OA), der Uhler Baggersee ist mit UB gekennzeichnet

Fig. 1 Geological units in the catchment area of the river Möhlin, sample locations as well as the courses of the rivers Möhlin, Ahbach, Eckbach, Neumagen and Seltenbach. The geology is based on a map from LUBW (2012). UM and OA represent the water gauges Untermünstertal and Oberambringen, the Uhler Gravel Pond is marked with UB

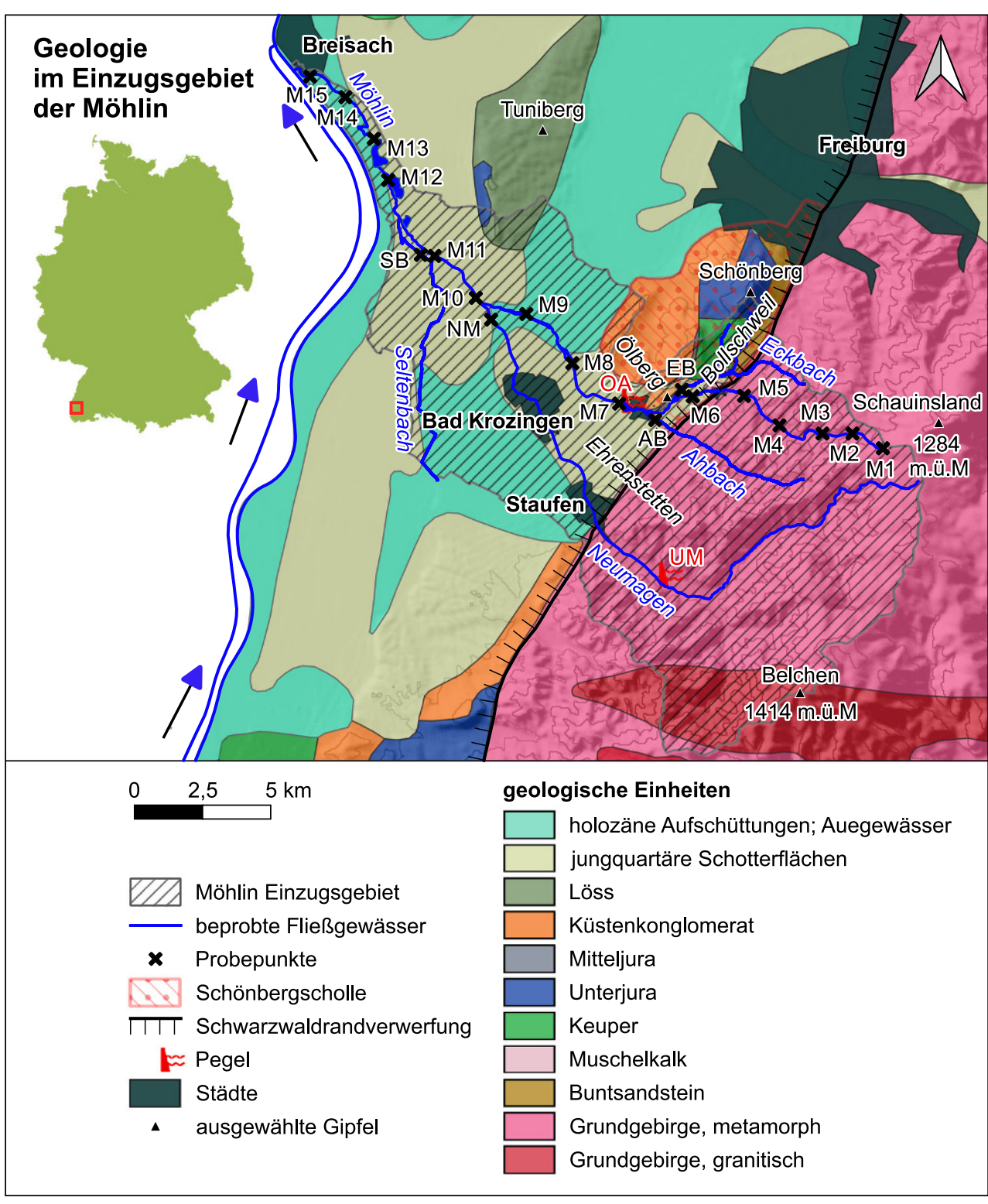

wasserspiegels und hat somit Auswirkungen auf das Fließund Infiltrationsverhalten sowie die Fließgeschwindigkeit des Flusses (Gallusser und Schenker 1992; Thiem 2006). Möhlin und Neumagen sind durch den sinkenden Grundwasserspiegel anfälliger für sommerliche Austrocknung, allerdings haben die Begradigungen der beiden Flüsse in der Vergangenheit auch zu verstärkten Hochwasserereignissen geführt (Thiem 2006). Die Flüsse weisen höhere Sensitivität gegenüber Wetterereignissen auf.

Die Möhlin entspringt dem kristallinen Grundgebirge des Schwarzwalds. Die hier anstehenden Gesteine sind äuBerst gering durchlässig, sodass der unterirdische Wasserabfluss auf Kluftvernetzungen als Leiter angewiesen ist. Zusammenhängende Aquifere sind aufgrund der Hanglage und der geringen Bodenmächtigkeit nicht zu erwarten.
Dies führt dazu, dass in diesen Hanglagen viele kleinere Punktquellen, darunter die Möhlinquelle sowie einige Zuflüsse, mit geringen Schüttungsraten sowie geringer Mineralisation aus dem oberen aufgelockerten Grundgebirge austreten (Plum et al. 2009; Geyer et al. 2011). Die beprobten Nebenflüsse Neumagen und Ahbach entspringen unter ähnlichen Bedingungen aus dem Hochschwarzwald, der Eckbach entspringt zu größeren Teilen aus dem Marktgräfler Land. Im Flussverlauf der Möhlin ändert sich der Untergrund, und das Grundgebirge des Schwarzwalds wird erst vom Marktgräfler Hügelland und im Anschluss von den Lockergesteinen des Oberrheingrabens abgelöst. $\mathrm{Ab}$ dem Eintritt ins Marktgräfler Hügelland, etwa auf der Höhe von Bollschweil, gelangt die Möhlin in das weitreichende Grundwasserleiter-System des Oberrheingrabens. In der 
Vorbergzone wird dieses dominiert durch stark verwittertes Sediment aus dem Hochschwarzwald, welches eine geringere hydraulische Durchlässigkeit als die alpinen Sedimente des mittleren Oberrheingrabens besitzt (Lang et al. 2005; Wirsing und Luz 2007). Der Aquifer weist hier Mächtigkeiten von einigen Metern bis zu etwa $50 \mathrm{~m}$ auf (Al Najem 2016). In den quartären Ablagerungen im Bereich des Rheins steigt die Durchlässigkeit an, da der Granit-Gneishaltige Feinkornanteil des Schwarzwalds von gröberem alpinen Material mit einem hohen Kalksteinanteil abgelöst wird (GLA Baden Württemberg 1979; Wirsing und Luz 2007). Der Aquifer des Oberrheingrabens, welcher einen der größten zusammenhängenden Aquifere Europas darstellt, erreicht in der Grabenmitte Mächtigkeiten von $200 \mathrm{~m}$.

Das Grundwasser fließt im Untersuchungsgebiet von Ost nach West in Richtung Rhein, wo es dann mit der Fließrichtung des Rheins nach Norden abbiegt. Die mit der Rheinbegradigung einhergehende Grundwasserspiegelabsenkung sorgt für die Infiltration von Flusswasser der Rheinvorfluter in den Aquifer, die im Extremfall zur Austrocknung von Flüssen wie der Möhlin oder der Dreisam in den Sommermonaten führen kann. Eine Exfiltration des Grundwassers ist in Rheinnähe zu beobachten. Der Rhein kann Exfiltrationen, sowie Infiltrationen bewirken. Im Untersuchungsgebiet sorgen Stauwehre für eine Infiltration von Rheinwasser vor Breisach (Bauer et al. 2005).

Im Untersuchungsgebiet weist das Grundwasser in tieferen Bereichen erhöhte Salinität mit $\mathrm{Cl}^{-}$-Konzentrationen bis in den Grammbereich auf (Bauer et al. 2005). Grund hierfür sind punktuelle Eintragungen durch Abraumhalden und Absatzbecken des Kalibergbaus im Gebiet des Elsass und auf deutscher Seite. Haupteinträge bilden die Absatzbecken rund um die Fessenheimer Insel, sowie die Abraumhalden bei Heitersheim und Buggingen (Bauer et al. 2005; Lang et al. 2005; Lucas et al. 2010).

\section{Geologie}

Das EZG der Möhlin unterteilt sich in zwei große Naturräume: Im Osten den Schwarzwald mit seinen Vorbergen und im Westen, bis hin zur Mündung in den Rhein, den Oberrheingraben (Geyer et al. 2011). Das EZG der Möhlin umfasst eine große Anzahl geologischer Grund- und Deckgebirgseinheiten, in denen sich mehr als 300 Mio. Jahre Erdgeschichte widerspiegeln (Abb. 1). Die Entstehung des Oberrheingrabens, welche ihre Anfänge im mittleren Eozän hatte (Schumacher 2002), sorgte für eine veränderte Entwässerung des westlichen Schwarzwalds, eine erhöhte Erosion des Hochgebirges und ein Abkippen des westlichen Schwarzwaldrandes um bis $\mathrm{zu} 60^{\circ}$, welches zur Herausbildung einer im Untersuchungsgebiet ca. $3 \mathrm{~km}$ breiten Vorbergzone, der Schönbergscholle, die Schichtenfolgen vom
Mittleren Buntsandstein bis hin zu pleistozänen Gesteinen enthält, geführt hat (Mauser 1974).

Das Quellgebiet der Möhlin wird geprägt durch variszische Migmatite und Paragneise (Geyer et al. 2011). Hauptminerale in den Migmatiten sind Quarz, Biotit, Feldspat, Cordierit und Sillimanit und in den Paragneisen Biotit, Quarz und Plagioklas (LGRB 2019). Im weiteren Verlauf durchläuft die Möhlin granitisches Gebirge, um sodann im oberen Mittellauf auf einer Fließstrecke von etwa $500 \mathrm{~m}$ Buntsandstein zu durchqueren. Das Flussbett selbst ist hier teilweise mit Auenlehm aufgefüllt. Der Eckbach, ein Nebenfluss der Möhlin, durchfließt Muschelkalk und Keuperschichten bevor er in die Möhlin mündet. Hinter Bollschweil trifft die Möhlin auf kalkarmen quartären Lösslehm aus dem Schwarzwald (Schreiner 1991; LGRB 2019). Nach Zufluss des Eckbachs umfließt die Möhlin am Westende der Schönbergscholle den Ölberg. Die Ostflanke des Ölbergs weist, von Ost nach West, Jura-Gesteine der Hauptrogenstein- und der Variansmergel-Formation auf. Den Großteil des Ölbergs bilden jedoch die sand- und mergelsteinhaltigen Blockkonglomerate und Konglomerate der tertiären Küstenkonglomerat-Formation.

Westlich von Ehrenstetten wandelt sich das Landschaftsbild und das Relief wird flacher. Prägendes geologisches Element sind hier quartäre Lössablagerungen. Der Löss im Oberrheingraben stammt größtenteils aus alpinen Gebieten (Schreiner 1991; LGRB 2019). Das Flussbett wird hier von einem etwa $10 \mathrm{~m}$ breiten Gürtel aus Hochflutlehm und Auensand umrandet, welcher aus abgelagerten Sedimenten der Möhlin besteht. In diesem Bereich fließt der Neumagen in die Möhlin.

Der Neumagen ist der abflussstärkste Nebenfluss der Möhlin und entspringt wie diese im Hochschwarzwald. Im Quellgebiet des Neumagens finden sich Paragneise, Migmatite und Granite sowie der Münstertal-Quarzporphyr, ein Zeuge des frühkarbonischen Vulkanismus (Geyer et al. 2011). Im Mittellauf durchfließt der Neumagen größere Teile der Neuenburg-Formation sowie Löss und Auensand. Die Gesteine der Trias und des Jura spielen mit Verbreitungsflächen von $<1 \mathrm{~km}^{2}$ eine untergeordnete Rolle.

Nach Zufluss des Neumagen fließt die Möhlin noch etwa $4 \mathrm{~km}$ durch Auenlehm, bevor sie in Rheinnähe wiederum auf die Neuenburg-Formation trifft. Diese ist hier durch ihre Nähe zum Rhein stark alpin geprägt und daher kalkhaltiger als am Grabenrand (LGRB 2019). Hier knickt die Möhlin $\mathrm{ab}$ und fließt parallel zum Rhein durch Auensedimente, bevor sie den Uhler Baggersee, eine aktive Kiesgrube, durchquert. Anschließend fließt die Möhlin durch von karbonatreichen Auenmergeln dominierte Rheinauen, welche vom Talauenschotter der Neuenburg-Formation durchzogen sind. 


\section{Methodik}

Die Auswahl der Probepunkte richtete sich nach der geologischen und hydrologischen Situation. Beprobt wurde in der Regel immer an der Grenze benachbarter Gesteinseinheiten, um deren Einflüsse zu erfassen, ohne die Einflüsse nachfolgender Formationen mit einzubeziehen. Nebenflüsse mit einem erhöhten Abflussbeitrag oder charakteristischer Geologie wurden ebenfalls beprobt. Um den hydrochemischen Einfluss der Nebenflüsse zu bestimmen, wurden Probepunkte unmittelbar vor den Zuflüssen, als auch einige hunderte Meter nach den Zuflüssen gewählt. Insgesamt ergaben sich somit 15 Probepunkte im Flussverlauf der Möhlin (Möhlin (M1-M15)) sowie 4 Proben von Zuflüssen (Eckbach (EB), Ahbach (AB), Neumagen (NM), Seltenbach (SB)) (Abb. 1).

$\mathrm{Da}$ in Niedrigwassersituationen Einflüsse der Geologie auf die Wasserchemie stärker zutage treten, als in Perioden mit erhöhtem Abfluss (McDiffet et al. 1989), wurde für die Beprobung ein Zeitraum mit möglichst geringem Abfluss gewählt. Die Probennahme erfolgte am 16.07.2019. Dieser Tag wies sehr geringe Bewölkung sowie hohe Außentemperaturen (bis $35^{\circ} \mathrm{C}$ ) nach einer längeren Trockenperiode auf. Die Proben wurden in den obersten $10 \mathrm{~cm}$ in Flussmitte unter Ausschluss von Sauerstoff entnommen. Temperatur und Leitfähigkeit wurden vor Ort gemessen. An den Lokalitäten M9 und M12 war keine Probennahme möglich, da der Fluss an diesen Stellen trockengefallen war. An den Lokalitäten M1, M3, M7, M13, M15 und NM wurden zusätzlich separate Proben zur Isotopenanalyse entnommen.

Schüttungs- und Abflussmessungen wurden an den Messstellen M1 und M3 mittels Gefäßmessung durchgeführt. Für M7 und den Zufluss Neumagen (NM) liegen stündlich gemessene Abflussdaten der Hochwasservorhersagezentrale Baden-Württemberg (HVZ-BW) vor, die aus Wasserstands-Durchfluss-Beziehungen ermittelt werden.

Die Alkalinität und der $\mathrm{pH}$-Wert sowie die Ionenkonzentrationen $\mathrm{Ca}^{2+}, \mathrm{Mg}^{2+}, \mathrm{Na}^{+}, \mathrm{K}^{+}, \mathrm{SO}_{4}{ }^{2-}, \mathrm{Cl}^{-}, \mathrm{F}^{-}, \mathrm{NO}_{3}{ }^{-}$wurden am Institut für Geo- und Umweltnaturwissenschaften, Universität Freiburg, ermittelt. Für die Hydrogencarbonatbestimmung wurde eine alkalimetrische Titration mit dem pH-Endpunkt 4,3 vorgenommen. Bei Proben mit einem pHWert $>8,2$ wurde eine 2 -fache Titration auf die $\mathrm{pH}-$ Werte 8,2 und 4,3 vorgenommen (Koelle 2010). Die Ionenkonzentrationen wurden nach vorheriger Filterung mit $0,45 \mu \mathrm{m}$ Spritzfiltern per Ionenchromatographie durch das DX-120 der Marke Dionex ermittelt. Die Analyse wurde mithilfe der Bestimmung der Elektroneutralität kontrolliert.

Die $\delta \mathrm{D}$ - und $\delta^{18} \mathrm{O}$-Isotopenanalysen erfolgten mittels $\mathrm{Ca}$ vity-Ring-Down-Spektroskopie (CRDS) am Institut für Hydrologie der Universität Freiburg. Die $1000 \mu l$-Wasserproben wurden jeweils ohne vorherige Filterung mithilfe des L2130-i Cavity-Ring-Down-Spektrometers der Marke Pi- carro analysiert. Die Genauigkeit für diese Methode lag für ${ }^{18} \mathrm{O} /{ }^{16} \mathrm{O}$-Verhältnisse bei $\pm 0,16 \%$ und für ${ }^{2} \mathrm{H} /{ }^{1} \mathrm{H}$-Verhältnisse bei $\pm 0,6 \%$.

Die Sättigung bezüglich Kalzit wurde mit dem Programm PHREEQC (Parkhurst und Appelo 1999) ermittelt. Das Modell basiert auf thermodynamischen Grundsätzen von Gleichgewichtsreaktionen, welche aus einer Datenbank ausgelesen werden (Moel et al. 2013). Grundlage der in dieser Studie durchgeführten Berechnungen war die Datenbank phreeqc.dat.

\section{Ergebnisse und Diskussion}

\section{Abfluss und Physikochemie}

Die Höhe des Abflusses der Möhlin ist entlang des Flusslaufs starken Schwankungen ausgesetzt (Abb. 2a). An der Quelle wurde die Schüttung der Möhlin zu 0,261/s bestimmt. Der Abfluss steigt im Bereich des Schwarzwalds durch kleine Zuflüsse und ein geringes $\mathrm{Maß}$ von Grundwasserexfiltration rasch an und erreicht nach einigen Kilometern sein vorläufiges Maximum am Probepunkt M7 von 601/s. Nach dem Übergang aus der Schwarzwälder Vorbergzone in den Oberrheingraben verringert sich der Abfluss durch Infiltration von Flusswasser in den deutlich höher durchlässigen Untergrund und die Möhlin trocknet etwa $2,5 \mathrm{~km}$ vor der Lokation M9 vollständig aus, was in heißen Sommermonaten durch die klimatischen und hydrogeologischen Bedingungen nicht ungewöhnlich ist (Zollinger und Mäckel 1989; Koeniger 2003a; Reif et al. 2013; Gem. Bad Krozingen 2019). Mit Zufluss des Neumagens gelangt ein hydrochemisch anders geprägtes Wasser in die Möhlin (NM und M10). Die Hochwasservorhersagezentrale Baden-Württemberg beziffert den Abfluss des Neumagen am Pegel Untermünstertal (UM, Abb. 1), einige Kilometer flussaufwärts der Lokation NM, am Untersuchungstag auf 3881/s. An der Einmündung in die Möhlin beträgt er jedoch nur noch ca. 1001/s. Somit ist auch dieser Zufluss von Infiltration ins Grundwasser betroffen. Trotz des Zuflusses des Neumagens und einiger weiterer kleiner Bäche, läuft die Möhlin etwa $1 \mathrm{~km}$ nach der Messstelle M11 erneut trocken. Der Ausfluss des Uhler Baggersees weist wieder einen deutlichen Abfluss auf. Dieser steigt in den nächsten Kilometern stetig an und liegt bei M15 bei mehreren Kubikmetern pro Sekunde. Angesichts der Nähe zum Rhein dürfte es sich hierbei im Wesentlichen um infiltriertes Rheinwasser nach Passage durch den Untergrund handeln. Unterschiede bei Wassertemperatur, elektrischer Leitfähigkeit und in der chemischen Zusammensetzung der Möhlin in diesem Flussabschnitt im Vergleich zum Rheinwasser lassen auf eine längere Verweilzeit des exfiltrierenden Grundwassers 

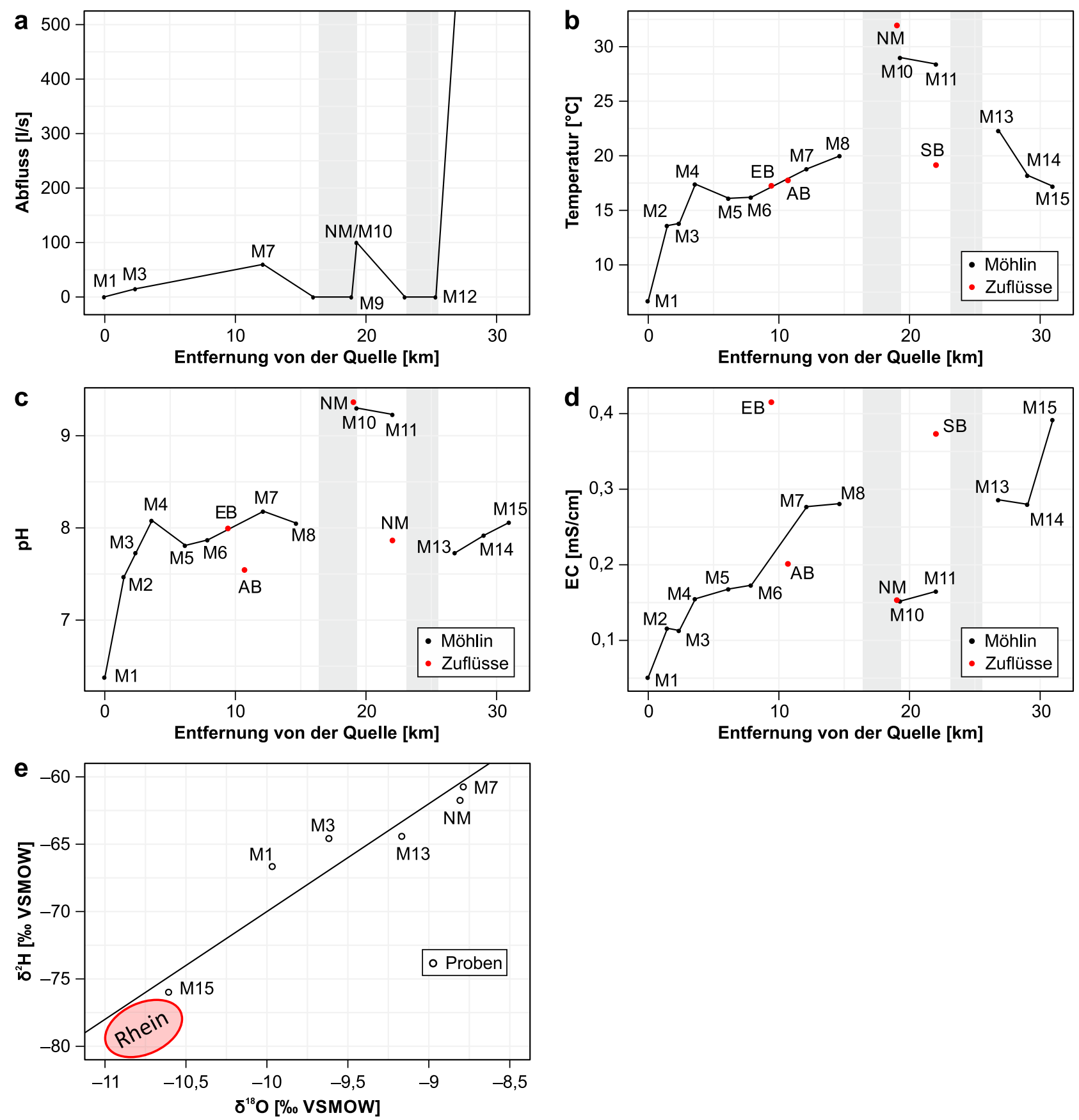

Abb. 2 Chemisch-physikalische Parameter sowie Isotope der Möhlin im Flussverlauf. Die roten Punkte stellen die Parameter der Zuflüsse dar. Im Bereich der grauen Flächen war der Flusslauf ausgetrocknet. Die eingezeichneten Rheinwerte stammen aus den Referenzen Koeniger und Leibundgut (2001) und Koeniger (2003b). Die Genauigkeit der Isotopenmessungen liegt für ${ }^{18} \mathrm{O} /{ }^{16} \mathrm{O}$-Verhältnisse bei $\pm 0,16 \%$ und für ${ }^{2} \mathrm{H} /{ }^{1} \mathrm{H}$-Verhältnisse bei $\pm 0,6 \%$

Fig. 2 Physio-chemical parameters in the course of the river Möhlin. The red dots represent the parameters of the inflows. In the gray area the river course was dried out. The shown Isotope data is mostly extracted (Koeniger und Leibundgut 2001; Koeniger 2003b). The accuracy of the Isotope measurements is at $\pm 0,16 \%$ for ${ }^{18} \mathrm{O} /{ }^{16} \mathrm{O}$ and $\pm 0,6 \%$ for ${ }^{2} \mathrm{H} /{ }^{1} \mathrm{H}$ 
mit geochemischer Interaktion im Untergrund schließen, bis es in die Möhlin gelangt.

Am Beprobungstag liegt die Wassertemperatur an der Quelle der Möhlin knapp unter $7^{\circ} \mathrm{C}$. Im Verlauf der Fließstrecke steigen die Temperaturen auf Werte über $20^{\circ} \mathrm{C}$ an (Abb. 2b). Die Wassertemperatur wird hauptsächlich durch die Sonneneinstrahlung auf das Gewässer und die Außentemperatur, welche unmittelbar mit der Tageszeit der Beprobung und der Höhenlage des Gewässers zusammenhängt, beeinflusst. Dass die flussaufwärts gelegenen Probepunkte früher am Tag beprobt wurden als die weiter flussabwärts gelegenen, begünstigt diesen Anstieg noch. Der Temperaturabfall zwischen den Lokationen M4 und M5 (Abb. 2b) ist durch eine hohe Beschattung in diesem Bereich zu erklären. Die Wassertemperatur des einfließenden Neumagen (Probe NM) ist mit $32{ }^{\circ} \mathrm{C}$ durch sein flaches, breites und weitgehend unbeschattetes Flussbett deutlich höher. Daher weist auch der nachfolgende Möhlinabschnitt (M10, M11) höhere Wassertemperaturen auf. Der Ausfluss des Uhler Baggersees (M13) hat wieder niedrigere Temperaturen von etwa $22^{\circ} \mathrm{C}$. Durch die Grundwasserzuflüsse ab M13 sinkt die Wassertemperatur deutlich unter die Temperatur des Rheins $\left(21,5^{\circ} \mathrm{C}\right.$, Palmrainbrücke, Weil am Rhein), was auf eine Abkühlung und somit eine längere Verweilzeit des Wassers im Untergrund hindeutet.

Der pH-Wert der Möhlin liegt mit zwei deutlichen Ausnahmen größtenteils um $\mathrm{pH}=8$ und somit an der Grenze von silikatischen zu kalksteingeprägten Einzugsgebieten (Abb. 2c) (Schneider et al. 2002). Von der Quelle bis zur Lokation M4 ist ein deutlicher Anstieg des pH-Werts zu verzeichnen, welcher auf die fehlende Alkalinität und Pufferkapazität der Möhlin an der Quelle zurückzuführen ist. Das Wasser tritt hier mit einem pH-Wert von 6,38 aus dem Boden aus, was aufgrund des sauren Ausgangsgesteins nicht ungewöhnlich ist (Probst et al. 1999). Nach dem Quellaustritt nimmt der $\mathrm{pH}$-Wert rasch auf $\mathrm{pH} \approx 8 \mathrm{zu}$ (Abb. $4 \mathrm{c}$ ), wodurch die Pufferkapazität der Möhlin steigt. Die Lokationen NM, M10 und M11 im mittleren Abschnitt weisen ungewöhnlich hohe $\mathrm{pH}$-Werte von >9,2 auf. Dies ist wahrscheinlich auf das Vorhandensein von Algen im Flussbett zurückzuführen, welche durch Photosynthese dem Wasser Kohlensäure entziehen und gleichzeitig Hydroxidionen freisetzen und somit den $\mathrm{pH}$-Wert um mehrere Einheiten anheben können (Stabel 1986; Rieser 2002). In Rheinnähe fallen die $\mathrm{pH}$-Werte wieder auf $\mathrm{pH} \approx 8 \mathrm{ab}$.

Die elektrische Leitfähigkeit (EC) als indirektes Maß für TDS steigt über den gesamten Flussverlauf der Möhlin an

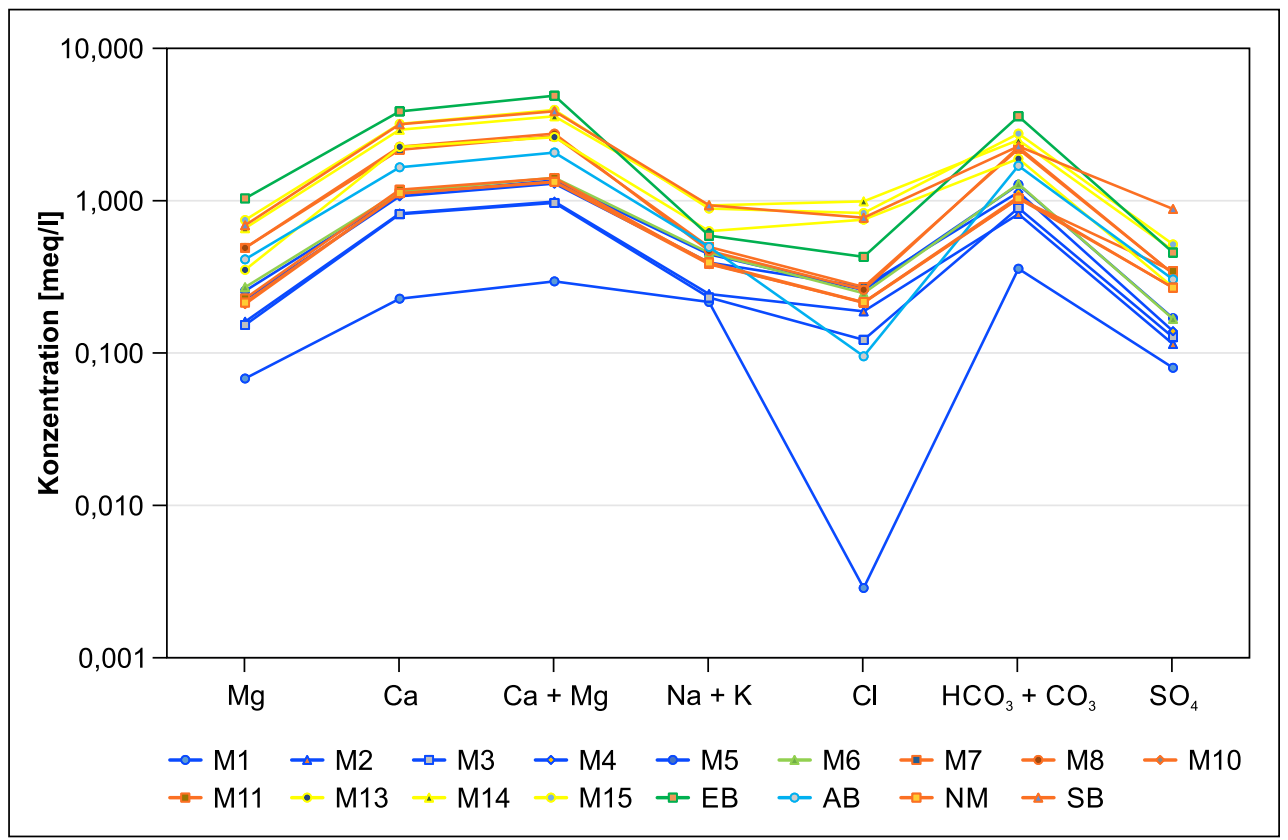

Abb. 3 Schoeller-Diagramm der untersuchten Wässer. Grundsätzlich steigt die Konzentration der Wässer mit der zurückgelegten Fließstrecke, relativ dazu nimmt die Chlorid-Konzentration zu. Eine Ausnahme stellt die Wasserprobe aus dem Eckbach (grün) mit sehr hohen Gesamtlösungsinhalten dar. Die Wässer aus dem kristallinen Grundgebirge (blau) weisen sehr niedrige Konzentrationen auf im Vergleich zu den Wasserproben in Schwarzwaldrandnähe (orange) und in Rheinnähe ( $\mathrm{gelb}$ ). Deutlich ist die Abnahme des Na/Cl-Verhältnisses in Rheinnähe ( $\mathrm{gelb}$ ) an der zunehmenden Verflachung der ( $\mathrm{Na}+\mathrm{K})$-Cl-Linie erkennbar

Fig. 3 Schoeller diagram of the investigated waters. Generally the concentration of the waters increases with an increasing flow path, relative to this, the chloride concentration increases. An exception is made with the water sample from the Eckbach (green) which has very high totaldissolved-solid contents. The waters from the crystalline basement (blue) have very low concentrations compared to the water samples near the edge of the Black Forest (orange) and near the Rhine (yellow). The decrease in the $\mathrm{Na} / \mathrm{Cl}$ ratio near the Rhine (yellow) is evident through the flattening of the $(\mathrm{Na}+\mathrm{K})-\mathrm{Cl}$ line 
(Abb. 2d). Die Werte reichen von $0,051 \mathrm{mS} / \mathrm{cm}$ bis 0,416 $\mathrm{mS} / \mathrm{cm}$. Der Eckbach (Probe EB) besitzt, durch das großflächige Vorhandensein von kalkhaltigen Trias-, Jura- und Tertiärgesteinen im EZG, eine deutlich erhöhte elektrische Leitfähigkeit, die für den Anstieg der Leitfähigkeit in der Möhlin (M7, M8) verantwortlich ist. Die elektrische Leitfähigkeit liegt bis zum Zufluss des Eckbachs im Bereich silikatisch geprägter Gewässer (Schneider et al. 2002), ist also niedrig und vergleichbar mit benachbarten Flüssen wie der Dreisam (Rieser 2002). Nach dem ersten Trockenfallen der Möhlin und dem Zufluss des Neumagen nimmt die elektrische Leitfähigkeit im Möhlin-Wasser stark ab (M10, M11), hervorgerufen durch den Zufluss des Neumagen. Auffallend ist auch die hohe EC der Probe SB. Diese Probe entstammt dem Seltenbach, dessen EZG geologisch größtenteils im Rheintal liegt. Hohe EC-Werte sind hier höchstwahrscheinlich auf anthropogenen Eintrag zurückzuführen (s. unten). Nach dem zweiten Trockenfallen der Möhlin und dem vorhergehenden Zufluss des Seltenbaches ist die EC wieder deutlich erhöht (Abb. 2d) und steigt bis zur Mündung in den Rhein an. Die Werte liegen im Bereich des Rheinwassers (300-400 $\mu \mathrm{S} / \mathrm{cm}$ (IKSR 2018)). Aufgrund der Mischung vom weniger stark mineralisierten Ausflusswasser des Uhler Baggersees mit den Grundwasserzuflüssen kann jedoch davon ausgegangen werden, dass letztere höher mineralisiert sind.

\section{Hydrochemie}

Die Möhlin durchfließt auf ihrer relativ kurzen Fließstrecke sehr unterschiedliche geologische Einheiten und die chemischen Daten zeigen, dass die Geologie des EZGs der Möhlin einen wichtigen Einfluss auf die Hydrochemie hat. Aufgrund der ansteigenden Leitfähigkeit und Ionenkonzentrationen sowie der anstehenden Geologie im Flussverlauf, ergibt sich im Verlauf der Möhlin eine Entwicklung von einem „silikatisch“ zu einem „karbonatisch“ geprägten Fluss (Tab. 1; Abb. 3).

Die $\mathrm{Ca}^{2+}$-Gehalte der Möhlin und ihrer Zuflüsse liegen zwischen 4,46 und 75,31 mg/l. Die $\mathrm{Ca}^{2+}$ - und $\mathrm{Mg}^{2+}$-Konzentrationen korrelieren positiv mit der elektrischen Leitfähigkeit (Abb. 4a), wobei die $\mathrm{Mg}^{2+}-$ Gehalte $(0,81-12,30 \mathrm{mg} / \mathrm{l})$ deutlich niedriger sind als die $\mathrm{Ca}^{2+}-$ Gehalte. Ein starker Anstieg der $\mathrm{Ca}^{2+}$ - und $\mathrm{Mg}^{2+}$-Konzentrationen ist am Ende der Fließstrecke der Möhlin im Bereich der Lokalitäten M13 $\left(\mathrm{Ca}^{2+}: 44,19 \mathrm{mg} / \mathrm{l}, \mathrm{Mg}^{2+}: 4,18 \mathrm{mg} / \mathrm{l}\right)$ bis $\mathrm{M} 15\left(\mathrm{Ca}^{2+}\right.$ : 62,50 mg/l, $\mathrm{Mg}^{2+}: 8,86 \mathrm{mg} / \mathrm{l}$ ) zu erkennen (Abb. 4a). Dementsprechend ist auch bei den $\mathrm{HCO}_{3}{ }^{-}$-Ionen ein genereller Anstieg der Konzentrationen von der Quelle bis zur Mündung zu erkennen (Abb. 4 b). Die Werte liegen zwischen 21,36 und $213,40 \mathrm{mg} / \mathrm{l}$.

Die gemessenen Häufigkeiten von $\mathrm{Ca}^{2+}, \mathrm{Mg}^{2+}-$ und somit auch die $\mathrm{HCO}_{3}{ }^{-}$-Konzentrationen im oberen Flusslauf (Lokationen M1-M8) sind geologischer Natur und entstammen im Falle von $\mathrm{Ca}^{2+}$ und $\mathrm{Mg}^{2+}$ im Wesentlichen Anor-

Tab. 1 Analyseergebnisse

Table 1 Analyzed results

\begin{tabular}{|c|c|c|c|c|c|c|c|c|c|c|c|c|c|c|c|}
\hline $\begin{array}{l}\text { Loka- } \\
\text { tion }\end{array}$ & $\begin{array}{l}\text { Breiten- } \\
\text { grad }\end{array}$ & $\begin{array}{l}\text { Längen- } \\
\text { grad }\end{array}$ & $\begin{array}{l}\text { ELF } \\
{[\mathrm{mS} / \mathrm{cm}]}\end{array}$ & $\mathrm{pH}$ & $\begin{array}{l}\text { Tempe- } \\
\text { ratur } \\
{\left[{ }^{\circ} \mathrm{C}\right]}\end{array}$ & $\begin{array}{l}\mathrm{Ca} \\
{[\mathrm{mg} / \mathrm{l}]}\end{array}$ & $\begin{array}{l}\mathrm{Mg} \\
{[\mathrm{mg} / \mathrm{l}]}\end{array}$ & $\begin{array}{l}\mathrm{Na} \\
{[\mathrm{mg} / \mathrm{l}]}\end{array}$ & $\begin{array}{l}\mathrm{K} \\
{[\mathrm{mg} / \mathrm{l}]}\end{array}$ & $\begin{array}{l}\mathrm{HCO}_{3} \\
{[\mathrm{mg} / \mathrm{l}]}\end{array}$ & $\begin{array}{l}\mathrm{CO}_{3} \\
{[\mathrm{mg} / \mathrm{l}]}\end{array}$ & $\begin{array}{l}\mathrm{SO}_{4} \\
{[\mathrm{mg} / \mathrm{l}]}\end{array}$ & $\begin{array}{l}\mathrm{Cl} \\
{[\mathrm{mg} / \mathrm{l}]}\end{array}$ & $\begin{array}{l}\mathrm{F} \\
{[\mathrm{mg} / \mathrm{l}]}\end{array}$ & $\begin{array}{l}\mathrm{NO}_{3} \\
{[\mathrm{mg} / \mathrm{l}]}\end{array}$ \\
\hline M1 & 47.9035 & 7.8714 & 0,05 & 6,38 & 6,7 & 4,5 & 0,8 & 4,4 & 0,7 & 21,4 & - & 3,8 & 0,1 & - & 5,7 \\
\hline M2 & 47.9078 & 7.8549 & 0,12 & 7,47 & 13,6 & 16,2 & 1,9 & 5,0 & 0,8 & 48,8 & - & 5,4 & 6,5 & - & 2,9 \\
\hline M3 & 47.9076 & 7.8430 & 0,11 & 7,73 & 13,8 & 16,0 & 1,8 & 4,7 & 0,8 & 53,4 & - & 6,0 & 4,2 & - & 3,3 \\
\hline M4 & 47.9063 & 7.8303 & 0,16 & 8,08 & 17,4 & 21,0 & 2,7 & 7,8 & 1,7 & 67,9 & - & 6,6 & 9,4 & - & 2,4 \\
\hline M5 & 47.9197 & 7.8071 & 0,17 & 7,81 & 16,1 & 21,6 & 3,0 & 9,0 & 1,8 & 76,0 & - & 8,0 & 9,0 & - & 1,8 \\
\hline M6 & 47.9215 & 7.7873 & 0,17 & 7,87 & 16,2 & 22,4 & 3,2 & 9,2 & 1,8 & 77,0 & - & 7,9 & 8,6 & - & 2,5 \\
\hline M7 & 47.9176 & 7.7387 & 0,28 & 8,18 & 18,8 & 42,2 & 5,8 & 9,8 & 2,4 & 130,2 & - & 15,6 & 9,4 & 0,08 & 5,9 \\
\hline M8 & 47.9306 & 7.7178 & 0,28 & 8,05 & 20,0 & 44,3 & 5,8 & 9,2 & 2,3 & 133,9 & - & 15,6 & 9,0 & 0,09 & 6,1 \\
\hline M9 & 47.9470 & 7.6875 & - & - & - & - & - & - & - & - & - & - & - & - & - \\
\hline M10 & 47.9510 & 7.6708 & 0,15 & 9,30 & 29,0 & 21,8 & 2,5 & 7,6 & 1,7 & 36,9 & 13,0 & 12,6 & 7,5 & 0,14 & 1,9 \\
\hline M11 & 47.9667 & 7.6443 & 0,17 & 9,23 & 28,4 & 23,2 & 2,7 & 7,7 & 1,7 & 35,9 & 12,5 & 16,2 & 7,4 & 0,14 & 1,3 \\
\hline M12 & 47.9667 & 7.6443 & - & - & - & - & - & - & - & - & - & - & - & - & - \\
\hline M13 & 48.0029 & 7.6189 & 0,29 & 7,73 & 22,3 & 44,2 & 4,2 & 12,9 & 2,2 & 112,2 & - & 12,7 & 26,1 & 0,13 & 2,2 \\
\hline M14 & 48.0145 & 7.6081 & 0,28 & 7,92 & 18,2 & 57,2 & 7,8 & 19,5 & 2,3 & 149,1 & - & 22,2 & 34,3 & 0,09 & 4,1 \\
\hline M15 & 48.0234 & 7.5875 & 0,39 & 8,06 & 17,2 & 62,5 & 8,9 & 18,6 & 2,3 & 164,2 & - & 24,3 & 28,8 & 0,09 & 4,2 \\
\hline EB & 47.9212 & 7.7719 & 0,42 & 8,00 & 17,3 & 75,3 & 12,3 & 11,7 & 2,6 & 213,4 & - & 21,5 & 14,8 & 0,11 & 9,4 \\
\hline $\mathrm{AB}$ & 47.9128 & 7.7565 & 0,20 & 7,55 & 17,8 & 32,4 & 4,9 & 10,1 & 1,9 & 101,0 & - & 14,3 & 3,3 & 0,13 & 4,4 \\
\hline NM & 47.9489 & 7.6731 & 0,15 & 9,37 & 32,0 & 21,9 & 2,6 & 7,8 & 1,8 & 32,3 & 14,4 & 12,7 & 7,5 & 0,16 & 1,8 \\
\hline SB & 47.9762 & 7.6321 & 0,37 & 7,87 & 19,2 & 62,1 & 8,2 & 18,8 & 3,7 & 135,5 & - & 41,4 & 26,8 & 0,19 & 13,2 \\
\hline
\end{tabular}




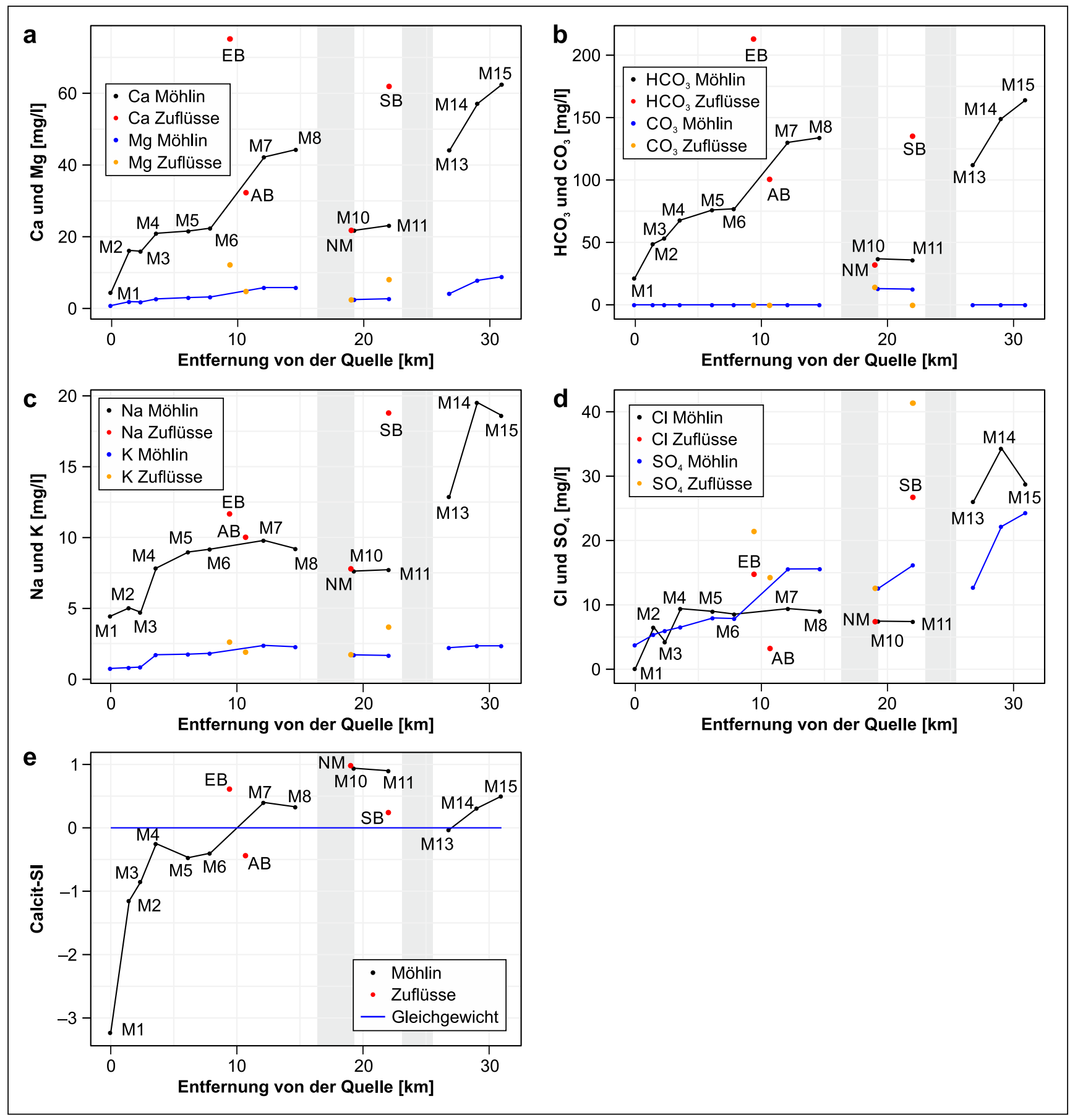

Abb. 4 Ionenkonzentrationen und Sättigung bezüglich Kalzits im Flussverlauf der Möhlin. Die roten und orangenen Punkte stellen die Ionenkonzentrationen der Zuflüsse dar. Im Bereich der grauen Flächen war der Flusslauf ausgetrocknet

Fig. 4 Ionic concentrations and calcite saturation in the course of the river Möhlin. The red and orange dots represent the ionic concentrations of the inflows. In the gray area the river course was dried out

thit-reichem Plagioklas, resp. Biotit, (Stober und Bucher 1999a). Der Gesamtlösungsinhalt ist an der Quelle aufgrund der silikatisch geprägten Gesteine, insbesondere im kristallinen Grundgebirge erwartbar gering und erhöht sich bis zum Probennahmepunkt M6 auf etwas über $20 \mathrm{mg} / \mathrm{l}$
(Abb. 4). Die Möhlin ist bis zur Lokation M7 an Kalzit untersättigt.

Die Konzentrationen der $\mathrm{Ca}^{2+}, \mathrm{Mg}^{2+}$ - und $\mathrm{HCO}_{3}-$-Ionen verdoppeln sich annähernd zwischen den Messstellen M6 und M7 (Abb. 4a,b), sodass erstmals eine leichte KalzitÜbersättigung im Wasser der Möhlin ab Messstelle M7 zu 
beobachten ist (Abb. 4e). Hier fließt die Möhlin durch die Schwarzwälder Vorbergzone, welche auch Trias-, Jura- und Tertiärgesteine enthält. Bedeutsam für die Hydrochemie ist die unmittelbare Nähe zum Ölberg, an dem Hauptrogenstein (Dogger) ansteht. Der Anteil kalkhaltiger Gesteine im direkten Umfeld der Möhlin ist somit erhöht, was den Anstieg der Konzentration erklärt. Gleichzeitig fließt hier der Eckbach hinzu, welcher fast ausschließlich die Gesteinseinheiten Muschelkalk, Keuper und Jura durchquert und die höchsten $\mathrm{Ca}^{2+}$, $\mathrm{Mg}^{2+}$ - und $\mathrm{HCO}_{3}^{-}$-Konzentrationen aller Proben aufweist (Abb. 3). Der Eckbach enthält generell hohe Ionenkonzentrationen und ist nach seinem Zufluss für einen Großteil der gelösten Stoffe der Möhlin bis zu ihrer ersten Austrocknung verantwortlich. Das Wasser im Eckbach ist bezüglich Kalzit stärker übersättigt als das Wasser in der Möhlin nach dem Zufluss des Eckbachs (M7).

Im Schöller-Diagramm (Abb. 3) hebt sich der Eckbach deutlich durch seine hohen Anionen- und Kationen-Konzentrationen von den anderen Messstellen ab. Die erhöhte Lösungsfracht entstammt nicht nur aus Kalzit-führenden Gesteinen, sondern wie die $\mathrm{SO}_{4}{ }^{2-}$-Konzentration zeigt, auch aus der Lösung von Gips, bzw. ggf. Anhydrit-Mineralen, die vor allem im Keuper aber auch im Muschelkalk zu finden sind (Koelle 2010). Verglichen mit den $\mathrm{Ca}^{2+}$-Konzentrationen weisen allerdings insbesondere die Messungen an den Probepunkten M11, SB aber auch NM und M10 erhöhte $\mathrm{SO}_{4}{ }^{2-}$-Gehalte auf (Abb. 3), was zumindest im Falle des Seltenbachs auf die Landwirtschaft zurückzuführen ist. Der in molaren Mengen fast identisch ablaufende Konzentrationsanstieg von $\mathrm{Ca}^{2+}$ - und $\mathrm{SO}_{4}{ }^{2-}$-Ionen (Korrelation: 0,99) zwischen den Lokationen NM und M11 ist wohl auf Gipsverwitterung zurückzuführen. Eine Erhöhung durch Abwassereinleitungen oder Düngung ist unwahrscheinlich, da andere Verbindungen, welche auf diese Eintragspfade in Gewässer gelangen könnten $\left(\mathrm{NO}_{3}^{-}, \mathrm{NaCl}, \mathrm{KCl}\right)$, nicht oder nur in geringen Konzentrationen vorliegen.

Mit dem Ahbach gelangt vor Messstelle M7 ein weiterer Zufluss mit einer leicht höheren $\mathrm{Ca}^{2+}$-Konzentration $(25 \mathrm{mg} / \mathrm{l})$ in die Möhlin, die jedoch nicht geologisch bedingt, sondern wahrscheinlich auf Waldkalkungen im Gebiet des Ahbachs zurückzuführen sind.

Die molare $\mathrm{Mg}^{2+}-$ Konzentration bewegt sich um den Faktor 0,16-0,27 der molaren $\mathrm{Ca}^{2+}$-Konzentration und liegt somit im zu erwartenden Bereich für Oberflächengewässer $(0,1-0,4)$ (Koelle 2010). Anthropogene Beeinflussungen sind nicht zu erkennen. Die $\mathrm{Mg}^{2+} / \mathrm{Ca}^{2+}$-Verhältnisse in der Möhlin sind grundsätzlich deutlich niedriger, sowohl auf ihrer Fließstrecke im kristallinen Grundgebirge als auch im Rheintal, im Vergleich zu denen des Eckbach und Abbach, deren Talsohlen stärker durch Dolomit-führende Gesteine geprägt sind. In den Gesteinen des Jura ist der $\mathrm{Mg}^{2+}$-Gehalt gering. Diese haben jedoch ein zu geringes Verbreitungsgebiet, um die $\mathrm{Mg}^{2+}-$ Gehalte der Möhlin signifikant zu be- einflussen und sind im Schoeller-Diagramm nur schwach erkennbar (Abb. 3).

Die $\mathrm{Na}^{+}$- und $\mathrm{K}^{+}$-Gehalte liegen zwischen 4,44 und 19,53 resp. 0,74-3,70 mg/l (Abb. 4c). Die niedrigsten Konzentrationen treten an der Möhlin-Quelle (M1) auf. Kalium verhält sich im Verlauf der Fließstrecke ähnlich dem $\mathrm{Na}^{+}$, hat jedoch aufgrund seiner hohen Reaktivität und daher geringeren freien Verfügbarkeit die zu erwartenden niedrigeren Konzentrationen (Abb. 4c). Eckbach (EB) und Ahbach (AB) weichen mit $\mathrm{Na}^{+}$-Werten von 11,70 und $10,05 \mathrm{mg} / \mathrm{l}$ und $\mathrm{K}^{+}$-Werten von 2,64 und $1,93 \mathrm{mg} / \mathrm{l}$ geringfügig von den $\mathrm{Na}^{+}$- und $\mathrm{K}^{+}-$Gehalten der Möhlin ab. Das in die Möhlin einmündende Wasser des Seltenbachs (Probe SB) besitzt mit $18,83 \mathrm{mg} / \mathrm{l} \mathrm{Na}^{+}$und $3,70 \mathrm{mg} / 1 \mathrm{~K}^{+}$vergleichsweise hohe Konzentrationen. Herausstechend ist der nach der Durchquerung des Uhler Baggersees (UB) steigende $\mathrm{Na}^{+}$Gehalt auf ein Maximum von 19,53 mg/l (M14), während die $\mathrm{K}^{+}$-Konzentration keinen vergleichbaren Anstieg aufweist (Abb. 4c).

Das molare $\mathrm{Na}^{+} / \mathrm{Cl}^{-}$-Verhältnis ist an der Quelle der Möhlin mit 68,4 sehr hoch, was darauf hinweist, dass das $\mathrm{Na}^{+}$durch Kationenaustauschprozesse im Boden ausgewaschen wurde und somit letztlich aus der Feldspatverwitterung (i.w. Albit) stammt. Die Auswaschung von „basischen“ Kationen $\left(\mathrm{Na}^{+}, \mathrm{K}^{+}, \mathrm{Ca}^{2+}, \mathrm{Mg}^{2+}\right)$ ist auf den sauren Böden des Schwarzwalds zu erwarten (Lazar 2005; Amelung et al. 2018). $\mathrm{Cl}^{-}$stammt dagegen in anthropogen unbelasteten Wässern im kristallinen Grundgebirge überwiegend aus der Verwitterung von Mineralen wie Biotit, Amphibol oder Apatit oder aus Flüssigkeitseinschlüssen bei der Alteration von Quarz (Stober und Bucher 1999b). Die Annahme, dass das Quellwasser stark von der Bodenlösung beeinflusst ist, wird durch den durch Nitrifikation im Boden erhöhten $\mathrm{NO}_{3}{ }^{-}$-Gehalt gestärkt. Der im Vergleich geringe $\mathrm{K}^{+}$-Gehalt kann durch stärkere Adsorption von $\mathrm{K}^{+}$ an die Bodenpartikel erklärt werden. Im weiteren Flussverlauf nimmt das $\mathrm{Na}^{+} / \mathrm{Cl}^{-}-$Verhältnis ab (molares $\mathrm{Na}^{+} / \mathrm{Cl}^{-}$Verhältnis: 1,6 (M7)), während weiterhin nur geringe $\mathrm{K}^{+}-$ Gehalte zu verzeichnen sind. An der Messstelle M4 in St. Ulrich ist ein sprunghafter Anstieg der $\mathrm{Na}^{+}$- und $\mathrm{Cl}^{-}$Gehalte zu verzeichnen, welcher wahrscheinlich auf den Zufluss des nicht beprobten Nesterbachs zurückzuführen ist. Der Nesterbach verläuft einige Kilometer neben einer stärker frequentierten Bergstraße (K4956) und wird im Winter durch Streusalz belastet.

Die $\mathrm{SO}_{4}{ }^{2-}$-Gehalte der Proben liegen zwischen 3,77 und $41,43 \mathrm{mg} / \mathrm{l}$. Nach dem Zufluss von Eckbach und Ahbach ist ein leichter Anstieg der $\mathrm{SO}_{4}{ }^{2-}$-Konzentration im Wasser der Möhlin zu verzeichnen (Abb. 4d). Im Oberlauf der Möhlin ist $\mathrm{SO}_{4}{ }^{2-}$ nur in sehr geringen Mengen gelöst (Abb. $4 \mathrm{~d}$ ). Der atmosphärische Sulfateintrag ist im Schwarzwald vergleichsweise gering (Mayer et al. 1995). Der $\mathrm{SO}_{4}{ }^{2-}$-Gehalt stammt daher höchst wahrscheinlich aus der Alteration von 
Sulfiden wie Pyrit, die im kristallinen Grundgebirge vorhanden sind (Stober und Bucher 1999b). Im weiteren Verlauf der Möhlin steigt die $\mathrm{SO}_{4}{ }^{2-}$-Konzentration leicht an, die Werte bewegen sich jedoch immer noch auf niedrigem Niveau. Höhere $\mathrm{SO}_{4}{ }^{2-}$-Werte (M8: 15,60 mg/l) werden erst nach Zufluss des Eckbachs erreicht.

Nach ihrer ersten Austrocknung, ab dem Zufluss des Neumagen hat das Möhlinwasser eine geringere $\mathrm{Ca}^{2+}$ Konzentration als davor (Lokationen NM-M11, <25 mg/l, Abb. 4a). Ursache dafür ist, dass der Neumagen in seinem Oberlauf durch silikatische Gesteine und im Unterlauf durch kalkarme Lössgebiete und die Neuenburg-Formation fließt. Die Neuenburg-Formation enthält hier aufgrund ihrer Nähe zum östlichen Rheingrabenrand verstärkt Material des kristallinen Grundgebirges und ist daher relativ arm an Karbonatgesteinen (LGRB 2019). Eine andere Ursache für die niedrige $\mathrm{Ca}^{2+}$-Konzentration im Möhlinwasser stellt die Übersättigung des Wassers bezüglich Kalzit dar $(\mathrm{SI} \approx 1, \mathrm{Abb} .4 \mathrm{e})$. Erhöhte SI-Werte sind auch durch hohe Wassertemperaturen zu erklären, die die Kalzit- und Kohlensäure-Löslichkeit verringern. Auch der Entzug von $\mathrm{CO}_{2}$ durch Algen im Flussbett kann dabei eine Rolle spielen. In diesem Flussabschnitt liegt ein Teil der gelösten Karbonate aufgrund des hohen $\mathrm{pH}$-Wertes als Karbonat-Ion $\left(\mathrm{CO}_{3}^{2-}\right)$ vor. Eine tatsächliche Ausfällung von Kalzit ist im Flussabschnitt zwischen den Lokationen NM und M11 jedoch nicht zu beobachten. Die Abnahme der Kalzit SIWerte zwischen den Lokationen NM und M11 (Abb. 4e) ist wahrscheinlich auf die sinkenden Wassertemperaturen in diesem Bereich zurückzuführen.

Im zweiten Flussabschnitt der Möhlin bleiben die $\mathrm{K}^{+}$, $\mathrm{Na}^{+}$- und $\mathrm{Cl}^{-}$-Gehalte weitgehend konstant (Abb. 4c, d). Das molare $\mathrm{Na}^{+} / \mathrm{Cl}^{-}$-Verhältnis beträgt 1,6. Da die Lösung von Halit $\mathrm{zu}$ einem $\mathrm{Na}^{+} / \mathrm{Cl}^{-}$-Verhältnis von 1 führt und da rezentes Meerwasser einen noch niedrigeren Wert aufweist, legt der höhere $\mathrm{Na}^{+} / \mathrm{Cl}^{-}$-Wert nahe, dass zumindest ein Teil der gelösten $\mathrm{Na}^{+}$-Ionen geologischen Ursprungs ist. Die zudem beobachtete leicht erhöhte $\mathrm{Cl}^{-}$-Konzentration (Abb. 3) stammt daher höchstwahrscheinlich aus kommunalen Abwässern oder Streusalz, wobei die Möhlin keine nennenswerte Belastung aufweist (nach LAWA 1998).

Fluorid ist erst ab Lokation M7 in nachweisbaren Mengen in der Möhlin vorhanden und zeigt sich in den restlichen Proben sehr konstant. Der Flussabschnitt des Neumagenwassers weist hohe $\mathrm{F}^{-}$-Konzentrationen im Flussverlauf der Möhlin auf. Insgesamt weist die Probe SB des Seltenbachs den höchsten $\mathrm{F}^{-}$-Gehalt auf $(0,19 \mathrm{mg} / \mathrm{l})$. Die Konzentrationen im Neumagenwasser könnten auf das Fluoritvorkommen im Grundgebirge und den alten Bergbau im Münstertal zurückzuführen sein, welches im EZG des Neumagen liegt. Das Münstertal ist reich an Flussspatvorkommen, welche im Bergwerk Teufelsgrund noch bis in das Jahr 1958 abgebaut wurden (Kuhn und Duba 2017).
Die Hydrochemie des letzten Flussabschnitts der Möhlin nach dem Ausfluss aus dem Uhler Baggersee (Lokation M13) bis zur Mündung in den Rhein bei Breisach (Lokation M15) ist vom Baggersee sowie von mehreren starken Grundwasserzuflüssen geprägt. Nach Ausfluss aus dem Uhler Baggersee weist die Möhlin hohe $\mathrm{Ca}^{2+}-$ und $\mathrm{Mg}^{2+}-\mathrm{Ge}-$ halte auf. Durch den weiteren Anstieg der Konzentrationen bis zur Mündung ist davon auszugehen, dass das zufließende Grundwasser für den zunehmenden Anstieg an $\mathrm{Ca}^{2+}$ und $\mathrm{Mg}^{2+}$ verantwortlich ist. Auch die Lösung dieser beiden Ionen aus den kalzithaltigen Auenmergeln mag einen Beitrag liefern. Die Möhlin weist zudem leicht erhöhte $\mathrm{HCO}_{3}{ }^{-}$-Konzentrationen auf sowie eine leicht zunehmende Sättigung bezüglich Kalzit. Die Konzentrationen übersteigen die aus dem Rheinwasser bekannten Werten.

Die $\mathrm{Na}^{+}$- und $\mathrm{Cl}^{-}$-Konzentration sind im Unterlauf der Möhlin ungewöhnlich hoch (Abb. 4c, d). Dies ist weitgehend eine Folge des Kalibergbaus im französischen Elsass sowie auf deutscher Seite (Bauer et al. 2005; Lang et al. 2005; Al Najem 2016). Der Kalisalzabbau wurde nahe Mulhouse für etwa 90 Jahre bis 2002 betrieben. Abwäs-

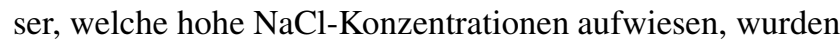
in den Rhein oder bei niedrigem Wasserstand in Lagerbecken geleitet, wodurch salzhaltiges Wasser ins Grundwasser gelangt ist (Lang et al. 2005). Dies hatte stark erhöhte $\mathrm{Cl}^{-}$-Konzentrationen im Rhein $\left(\mathrm{Cl}^{-}>100 \mathrm{mg} / \mathrm{l}\right)$ und dem benachbarten Grundwasser $\left(\mathrm{Cl}^{-}>140 \mathrm{mg} / \mathrm{l}\right)$ zur Folge (Bauer et al. 2005; IKSR 2018; bnNETZE GmbH 2019). Während die Konzentrationen im Rhein mittlerweile zurückgegangen sind, sind die $\mathrm{Cl}^{-}$-Konzentrationen im Grundwasser beständiger und beeinflussen mittlerweile die Breisacher Trinkwasserbrunnen. Der erhöhte Salzgehalt im Grundwasser wirkt sich durch die Grundwasserzuflüsse zwischen dem Uhler Baggersee und Breisach auch auf die Möhlin aus. Aufgrund der Verdünnung durch das Rheinuferfiltrat erreichen die Konzentrationen in der Möhlin jedoch nur Maximalwerte von 34,30 mg/l (Cl-, M14) und 19,53 mg/l $\left(\mathrm{Na}^{+}\right.$, M14). Dagegen weisen die $\mathrm{K}^{+}-$Gehalte, welche in Kaliabwässern stark erhöht sind, keine anormalen Werte auf. Bauer et al. (2005) und Lucas et al. (2010) führen dies auf Ionenaustauschprozesse im Grundwasser zurück. Nach dieser Studie sind Grundwasserproben, je weiter sie vom Eintragsort entfernt sind, überproportional an $\mathrm{K}^{+}$verarmt, während die $\mathrm{Cl}^{-}$-Konzentration hauptsächlich von Verdünnungsprozessen gesteuert werden. Im Falle der Grundwasserzuflüsse der Möhlin erklärt dies auch die erhöhten Werte von $\mathrm{Ca}^{2+}$ und $\mathrm{Mg}^{2+}$, welche anstelle von $\mathrm{K}^{+}$in Lösung gegangen sind.

Der $\mathrm{SO}_{4}{ }^{2-}$-Gehalt steigt im letzten Flussabschnitt der Möhlin (Lokationen M13-M15) noch einmal an (Abb. 4d), wobei der Ausfluss des Uhler Baggersees einen vergleichsweise geringen $\mathrm{SO}_{4}{ }^{2-}-$ Gehalt aufweist (Abb. 4d). Aufgrund des niedrigen $\mathrm{NO}_{3}{ }^{-}$-Werts (Lokation M13: 2,2 mg/l) fungiert der Uhler Baggersee als Nährstoffsenke. Grund für 
den anschließenden Anstieg der $\mathrm{SO}_{4}{ }^{2-}$-Konzentrationen ist höchstwahrscheinlich das Uferfiltrat des Rheins, welches als Grundwasseraustritt in die Möhlin fließt. Die $\mathrm{SO}_{4}{ }^{2-}$ Konzentration liegt hier bei $25 \mathrm{mg} / \mathrm{l}$, was in etwa den Mittelwerten des Rheins bei Weil am Rhein entspricht (IKSR 2018). Auch Grundwasserproben in diesem Gebiet in einem Radius von ca. $5 \mathrm{~km}$ zeigen $\mathrm{SO}_{4}{ }^{2-}$-Gehalte von etwa $30 \mathrm{mg} / \mathrm{l}$ (Bauer et al. 2005). Diese Konzentrationen können entweder durch Verwitterung aus den Alpenschottern der Neuenburg-Formation stammen, sie können jedoch auch eine Folge des Kalibergbaus sein.

\section{Isotopenzusammensetzung}

Die Möhlin zeigt am Anfang ihrer Fließstrecke die typische Wasserstoff- und Sauerstoff-Isotopie eines Fließgewässers mit steiler Talfahrt (Abb. 2e). Die von der Lokation M1 bis zur Lokation M7 stattfindende Anreicherung schwerer Wasserstoff- und Sauerstoff-Isotope ist durch den Höheneffekt des Niederschlags zu erklären. Für diesen werden in den Niederschlägen $\delta^{18} \mathrm{O}$-Abnahmen von $0,15-0,5 \%$ pro $100 \mathrm{~m}$ erwartet (Clark und Fritz 1997). Beim Vergleich der Isotopenwerte für Niederschlag in Freiburg (Ø (1999-2000) $\delta^{18} \mathrm{O}:-8,3$ (Bauer et al. 2005)) und den gemessenen Werten für die Quelle $\left(\delta^{18} \mathrm{O}\right.$ : $\left.-9,96\right)$ liegen diese, auch da Niederschläge im Sommer aufgrund höherer Temperaturen und somit geringerer Fraktionierung reicher an schweren Isotopen sind als im Winter (Dansgaard 1964), im erwarteten Rahmen. Darüber hinaus zeigt der Höheneffekt auf, dass sich die Möhlin aus vielen kleineren Zusammenflüssen zusammensetzt, was typisch für Gewässer aus dem GneisGranit-Komplex des Schwarzwalds ist, wo vorrangig Quellen mit kleinen Einzugsgebieten und geringen Schüttungen vorherrschen. Die Anreicherung schwerer Isotope in Probe M7 ist wahrscheinlich auf Verdunstungsprozesse zurückzuführen. Beim Verdampfen reichern sich leichte Isotope im Dampf an und das zurückbleibende Wasser erlangt eine schwerere Isotopensignatur. Ein solcher Effekt ist auch im Neumagen zu beobachten, welcher einige Kilometer flussabwärts einen ähnlichen Wert erreicht wie die Möhlin an der Messstelle M7 (Abb. 2e).

Das Wasser am Ausfluss des Uhler Baggersees (Lokation M13) ist leichter als die Wässer von Möhlin und Neumagen, jedoch deutlich schwerer als Rheinwasser bei Breisach (Koeniger und Leibundgut 2001; Koeniger 2003b). Daraus kann man entnehmen, dass Rheinuferfiltrat nur einen geringen Bestandteil des Uhler Baggersees ausmacht. Der erhöhte Deuterium-Excess-Wert der Probe M13 legt eine geringfügige Evaporation nahe. Möglich wären Mischungen aus lokalem Niederschlag, Möhlinwasser, Rheinuferfiltrat und Grundwasser aus lokaler Bildung.

Da sich die Isotopenzusammensetzung der Probe M15 nur geringfügig von der des Rheinwassers unterscheidet
(Koeniger und Leibundgut 2001; Koeniger 2003b), ist davon auszugehen, dass das infiltrierende Grundwasser zwischen den Messstellen M13 und M15 zu sehr großen Teilen aus Rheinwasser besteht. Die etwas schwerere Isotopenzusammensetzung der Probe M15 kann durch geringen Anteil an lokalem Grundwasser erklärt werden, jedoch sollte in diesem Bereich der Anteil an Rheinwasser bei etwa 70-90\% liegen. Grund für den Zufluss von Rheinwasser waren die hohen Abflusswerte im Juli 2019 in Kombination mit der Aufstauung des Wassers durch ein Stauwehr kurz vor der Möhlinmündung (Bauer et al. 2005). Dies sorgte für einen erhöhten Wasserspiegel im Rhein, welcher das Wasser dazu veranlasste ins Ufer zu infiltrieren. Die gemessenen $\delta \mathrm{D}-$ und $\delta^{18} \mathrm{O}-$ Isotopenzusammensetzungen sind in Abb. $2 \mathrm{e}$ gegen die GMWL dargestellt. Die $\delta D-$ und $\delta{ }^{18} \mathrm{O}$-Gehalte steigen im Flussverlauf bis zur Lokation M13 an. Der Neumagen (Lokation NM) weist mit der Möhlin vergleichbare Isotopenwerte auf. Die Wasserproben M13 und M15 sind isotopisch leichter als Proben der vorherigen Flussabschnitte, wobei M15 große Ähnlichkeiten mit im Rhein gemessenen Isotopenwerten aufweist (Koeniger und Leibundgut 2001; Koeniger 2003b).

\section{Schlussfolgerungen}

Die Möhlin weist im Oberlauf Eigenschaften eines grobmaterialreichen, silikatischen Mittelgebirgsflusses auf (Kriterien der Landesarbeitsgemeinschaft Wasser). Die chemische Zusammensetzung des Möhlinwassers wird im Oberlauf durch die geochemischen Eigenschaften der Granite und Gneise des Hochschwarzwalds geprägt (Abb. 5). Mit dem Zufluss von Ahbach und Eckbach und dem Erreichen von kalkreicherem Gestein verändert sich die chemische Zusammensetzung der Möhlin signifikant. Sie befindet sich in diesem Flussabschnitt im Grenzbereich von silikatisch und karbonatisch geprägten Wässern (Abb. 5). Mit dem Zufluss des Neumagen, welcher wenig karbonathaltiges Wasser enthält, wird der Effekt der karbonatischen Vorbergzone südlich von Freiburg wieder abgeschwächt. In ihrem Unterlauf wird die Möhlin in ihrer chemischen Zusammensetzung vom Uhler Baggersee, den sie durchläuft, beeinflusst, bevor diese Zusammensetzung nach und nach von der Wasserchemie des Rheins und im geringeren Maße durch anthropogene Kalibergbauverunreinigungen beeinflusst wird.

Die Wasserchemie der Möhlin wird maßgeblich durch die Geologie ihres EZGs geprägt. Aufgrund der zahlreichen Zuflüsse, welche entscheidend zum Abfluss der Möhlin beitragen, sind die verschiedenen Aspekte der Geologie im EZG der Möhlin sehr ausgeprägt in der chemischen Zusammensetzung des Wassers zu beobachten. Die extreme Niedrigwassersituation im Untersuchungszeitraum hat die unterschiedlichen Zusammensetzungen der Zuflüsse und den 
Abb. 5 Schematische Darstellung der Messungsergebnisse und deren Interpretation. Gezeigt ist die naturräumliche Gliederung nach der Landesanstalt für Umwelt BadenWürttemberg (LUBW). Die Diagramme stellen die prozentuale meq der gemessenen Ionen dar. Die verschiedenen Größen der Diagramme spiegeln die Verhältnisse der meq TDSGesamtkonzentrationen wieder. Die Grundwasserflüsse der Möhlin und ihrer Nebenarme entstammen eigenen Beobachtungen, während die Grundwasserflüsse des Rheins aus Bauer et al. (2005) entnommen wurden Fig. 5 Schematic representation of the measured results and their interpretation. Shown are the natural areas classified by the State Institute for the Environment Baden-Württemberg (LUBW). The diagrams show the percentage meq of the measured ions. The different sizes of the diagrams reflect the ratios of the total meq TDS-concentrations. The groundwater flows of the Möhlin and its tributaries come from our own observations, while the groundwater flows of the Rhine are partially taken from Bauer et al. (2005)

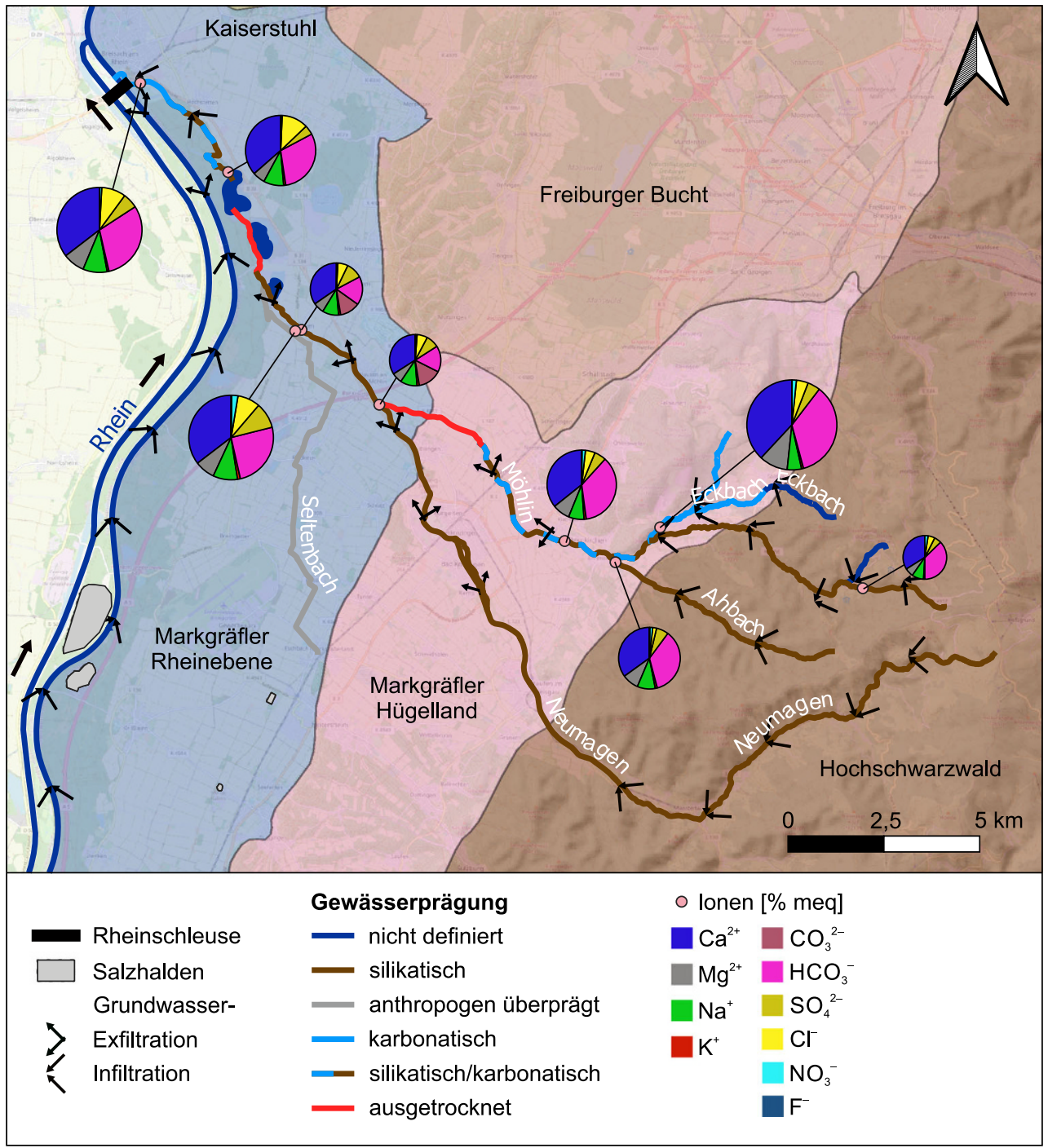

geologischen Hintergrund in der Wasserchemie der Proben um ein Vielfaches verstärkt. Die Isotopenuntersuchungen unterstützen die hydrochemischen Befunde.

Eine generelle Quantifizierung der Einflüsse der verschiedenen Wässer war aufgrund der Austrocknungen nicht möglich. Bei größeren Abflussereignissen würden jedoch vermutlich die silikatischen Prägungen der beiden Hauptarme Möhlin und Neumagen die Wasserchemie prägen.

Die vorliegende Untersuchung stellt eine Momentaufnahme dar und enthält somit keine zeitunabhängigen Informationen zur Wasserchemie der Möhlin. Der hydrologische und klimatologische Rahmen sowie die anthropogenen Stoffeinträge sind im Gegensatz zu den geologischen Rahmenbedingungen sehr variabel. Um diese Parameter genauer zu charakterisieren, müsste eine längerfristige Beprobung des Flusssystems für verschiedene Abflussverhältnisse und zu verschiedenen Jahreszeiten erfolgen.
Danksagung Wir bedanken uns bei zwei anonymen Reviewern für die konstruktive Diskussion und für wertvolle Anmerkungen. Des Weiteren bedanken wir uns bei Frau Sigrid Hirth-Walther sowie Frau Dr. Barbara Herbstritt für die Unterstützung in den Laboren.

Funding Open Access funding enabled and organized by Projekt DEAL.

Open Access Dieser Artikel wird unter der Creative Commons Namensnennung 4.0 International Lizenz veröffentlicht, welche die Nutzung, Vervielfältigung, Bearbeitung, Verbreitung und Wiedergabe in jeglichem Medium und Format erlaubt, sofern Sie den/die ursprünglichen Autor(en) und die Quelle ordnungsgemäß nennen, einen Link zur Creative Commons Lizenz beifügen und angeben, ob Änderungen vorgenommen wurden.

Die in diesem Artikel enthaltenen Bilder und sonstiges Drittmaterial unterliegen ebenfalls der genannten Creative Commons Lizenz, sofern sich aus der Abbildungslegende nichts anderes ergibt. Sofern das betreffende Material nicht unter der genannten Creative Commons Lizenz steht und die betreffende Handlung nicht nach gesetzlichen Vorschriften erlaubt ist, ist für die oben aufgeführten Weiterverwendungen des Materials die Einwilligung des jeweiligen Rechteinhabers einzuholen. 
Weitere Details zur Lizenz entnehmen Sie bitte der Lizenzinformation auf http://creativecommons.org/licenses/by/4.0/deed.de.

\section{Literatur}

Al Najem, S.: Hydrogeochemische Charakterisierung von Grundwässern des Oberrheingrabens zur Identifizierung störungsbedingter Tiefenwasser-Einflüsse. Dissertation. Ruprecht-Karls-Universität, Heidelberg (2016)

Albrecht, M.: Entwicklung der Nitratbelastung im Rohwasser des Wasserwerks Hausen a. d. Möhlin - eine hydrologisch-modelltechnische Untersuchung. Diplomarbeit. Albert-Ludwigs Universität, Freiburg (2006)

Amelung, W., Blume, H.-P., Fleige, H., Horn, R., Kandeler, E., KögelKnabner, I., Kretzschmar, R., Stahr, K., Wilke, B.-M.: Scheffer/ Schachtschabel Lehrbuch der Bodenkunde. Springer, Berlin, Heidelberg (2018)

Bauer, M., Eichinger, L., Elsass, P., Kloppmann, W., Wirsing, G.: Isotopic and hydrochemical studies of groundwater flow and salinity in the Southern Upper Rhine Graben. Int. J. Earth Sci. 94(4), 565-579 (2005). https://doi.org/10.1007/s00531-005-0500-5

bnNETZE GmbH: Trinkwasseranalyse Breisach (2019). https:// wasser.bnnetze.de/web/Downloads/Wasserversorgung/Breisach/ Trinkwasseranalyse-Breisach.pdf. Zugegriffen: 28. Aug. 2020

Clark, I.D., Fritz, P.: Environmental Isotopes in Hydrogeology. Lewis, Boca Raton (1997)

Dansgaard, W.: Stable isotopes in precipitation. Tellus 16(4), 436-468 (1964). https://doi.org/10.3402/tellusa.v16i4.8993

DWD: Niederschlag: vieljährige Mittelwerte 1981-2010 (2019). https://www.dwd.de/DE/leistungen/klimadatendeutschland/mittel werte/nieder_8110_akt_html.html?view=nasPublication\&nn= 480164. Zugegriffen: 27. Aug. 2020

Gallusser, W.A., Schenker, A. (Hrsg.): Die Auen am Oberrhein/Les zones alluviales du Rhin supérieur. Birkhäuser, Basel (1992). Ausmaß und Perspektiven des Landschaftswandels am südlichen und mittleren Oberrhein seit 1800/Etendue et perspectives de l'évolution des paysages dans le secteur méridional et moyen du Rhin supérieur depuis 1800

Gem. Bad Krozingen: Gewässer Bad Krozingen (2019). https://www. bad-krozingen.de/gewaesser, Zugegriffen: 25. Okt. 2019

Geyer, O.F., Gwinner, M.P., Simon, T.: Geologie von Baden-Württemberg, 5. Aufl. Schweizerbart, Stuttgart (2011)

GLA Baden Württemberg (Hrsg.): Hydrogeologische Karte von Baden-Württemberg, Oberrheingebiet, Freiburger Bucht mit Erläuterungen. Geologisches Landesamt und Landesanstalt für Umweltschutz Baden-Württemberg, Freiburg i. Brsg., Karlsruhe (1979)

IKSR: Vieljährige Jahresmittelwerte: Jahresmittelwerte verschiedener Parameter der Messstation Weil am Rhein (2018). http://iksr.bafg. de/iksr/lj_auswahl.asp?S=0, Zugegriffen: 23. Okt. 2019

Koelle, W.: Wasseranalysen - richtig beurteilt. Wiley-VCH, Hoboken (2010)

Koeniger, P. (Hrsg.): Tracerhydrologische Ansätze zur Bestimmung der Grundwasserneubildung. Freiburger Schriften zur Hydrologie, Bd. 16. Inst. für Hydrologie, Freiburg i. Brsg. (2003a)

Koeniger, P.: Tracerhydrologische Ansätze zur Bestimmung der Grundwasserneubildung. Albert-Ludwigs Universität, Freiburg (2003b)

Koeniger, P., Leibundgut, C.: Study of river water impacts on groundwater during flood events in a dry flood plain of the upper Rhine valley. In: Griebler C., Danielopol D.L., Gibert J., Nachtnebel H.P., Notenboom J. (Hrsg.): Groundwater ecology. Office for official publications of European Communities, Luxembourg (2001)

Kuhn, K., Duba, J. (Hrsg.): Fluss- und Schwerspat in Deutschland, S. 72. Bundesanstalt für Geowissenschaften und Rohstoffe (BGR), Hannover (2017)
Landesanstalt für Umwelt Baden-Würrtemberg: Hochwasservorhersagezentrale Baden-Württemberg: Möhlinpegel Oberambringen (2019). https://hvz.lubw.baden-wuerttemberg.de/pegel.html? id=00183, Zugegriffen: 22. Okt. 2019

Lang, U., Gudera, T., Elsass, P., Wirsing, G.: Numerical modelling of chloride propagation in the quaternary aquifer of the southern Upper Rhine Graben. Int. J. Earth Sci. 94(4), 550-564 (2005). https:// doi.org/10.1007/s00531-005-0477-0

LAWA: Beurteilung der Wasserbeschaffenheit von Fließgewässern in der Bundesrepublik Deutschland - chemische Gewässergüteklassifikation, 1. Aufl. Konzepte und Strategien Oberirdische Gewässer, Bd. 34. Kulturbuchverl., Berlin (1998)

Lazar, S.: Bodenzustandsbericht Baar, 1. Aufl. Bodenschutz, Bd. 19. LfU, Karlsruhe (2005)

LGRB: Geologie: Schichtenfolge, Lithologie, Zusammensetzung (2019). https://lgrbwissen.lgrb-bw.de, Zugegriffen: 7. Okt. 2019

Lucas, Y., Schmitt, A.D., Chabaux, F., Clément, A., Fritz, B., Elsass, P., Durand, S.: Geochemical tracing and hydrogeochemical modelling of water-rock interactions during salinization of alluvial groundwater (Upper Rhine Valley, France). Appl. Geochem. 25(11), 1644-1663 (2010). https://doi.org/10.1016/j.apgeochem. 2010.08.013

Mauser, P.F.: Eine späteiszeitliche Freilandstation auf dem Steinberg bei Bollschweil, LKR. Breisgau-Hochschwarzwald. Fundberichte aus Baden-Württemberg, Bd. 1. Schweizerbart, Stuttgart, S. 37-44 (1974)

Mayer, B., Feger, K.H., Giesemann, A., Jäger, H.-J.: Interpretation of sulfur cycling in two catchments in the Black Forest (Germany) using stable sulfur and oxygen isotope data. Biogeochemistry 30(1), 31-58 (1995). https://doi.org/10.1007/BF02181039

McDiffet, W.F., Beidler, A.W., Dominick, T.F., McCrea, K.D.: Nutrient concentration-stream discharge relationships during storm events in a first-order stream. Hydrobiologia 179, 97-102 (1989)

de Moel, P.J., van der Helm, A.W.C., van Rijn, M., van Dijk, J.C., van der Meer, W.G.J.: Assessment of calculation methods for calcium carbonate saturation in drinking water for DIN 38404-10 compliance. Drink. Water Eng. Sci. Discuss. 6(2), 167-198 (2013). https://doi.org/10.5194/dwesd-6-167-2013

Nitzsche, N.: Geologische, hydrogeologische und anthropogene Einflüsse auf die Wasserchemie der Möhlin. Bachelorarbeit. AlbertLudwigs Universität, Freiburg (2019)

Parkhurst, D.I., Appelo, C.A.J.: User's Guide to Phreeqc (Version 2) - a Computerprogram for Speciation, Batch-Reaction, OneDimensional Transport and Inversegeochemical Calculations. U.S. Geological Survey, Denver, S. 99-4259 (1999)

Plum, H., Dietze, G., Armbruster, V., Wirsing, G.: Natürliche geogene Grundwasserbeschaffenheit in den hydrogeochemischen Einheiten von Baden-Württemberg. LGRB, Freiburg i. Brsg. (2009)

Probst, A., Party, J.P., Fevrier, C., Dambrine, E., Thomas, A.L., Stussi, J.M.: Evidence of springwater acidification in the Vosges mountains (North-East of France): influence of bedrock buffering capacity. Water Air Soil Pollut. 114, 395-411 (1999)

Reif, A., Gärtner, S., Zimmermann, R., Späth, V., Lange, J.: Auenentwicklung am südlichen Oberrhein - „Trockenaue“ und rezente Rheinaue. Tuexenia 6, 125-169 (2013). Beiheft

Rieser, M.: Experimentelle Untersuchungen zur immissionsorientierten Bewertung der Wirkung von Niederschlagswassereinleitungen auf ein urbanes Gewässer am Beispiel der Dreisam im Stadtgebiet von Freiburg. Diplomarbeit. Albert-Ludwigs-Universität, Freiburg (2002)

Schneider, P., Neitzel, P.I., Schaffrath, M., Schlumprecht, H.: Leitbildorientierte physikalisch-chemische Gewässerbewertung - Referenzbedingungen und Qualitätsziele. In: Report R\&D-Project of the Environmental Federal Agency of Germany (Proj.-No. 20024 226). Umweltbundesamt, Chemnitz-Bayreuth (2002)

Schreiner, A.: Geologie und Landschaft. In: Hoppe, A. (Hrsg.) Marktgräflerland - Entwicklung und Nutzung einer Landschaft Berich- 
te der Naturforschenden Gesellschaft Freiburg, Bd. 81, S. 7-24. (1991)

Schumacher, M.E.: Upper Rhine Graben: role of preexisting structures during rift evolution. Tectonics 21(1), 6-1-6-17 (2002). https:// doi.org/10.1029/2001TC900022

Seidel, J., Mäckel, R.: Holocene sediment budgets in two river catchments in the southern upper Rhine valley, Germany. Geomorphology 92(3/4), 198-207 (2007). https://doi.org/10.1016/j. geomorph.2006.07.041

Stabel, H.H.: Calcite precipitation in Lake Constance: chemical equilibrium, sedimentation, and nucleation by algae 1 . Limnol. Oceanogr. 31(5), 1081-1094 (1986)

Stober, I., Bucher, K.: Deep groundwater in the crystalline basement of the Black Forest region. Appl. Geochem. 14, 237-254 (1999a)

Stober, I., Bucher, K.: Origin of salinity of deep groundwater in crystalline rocks. Terra Nova 11, 181-185 (1999b)
Thiem, K.: Die historische Landschaftsanalyse als Methode für die Fließgewässerbewertung am Beispiel des Münstertals im Schwarzwald. Inst. für Landespflege, Freiburg Breisgau (2006). Zugl.: Freiburg (Breisgau), Univ., Diss., 2005. VIII, 185, [20] S. Culterra, Bd. 46

Wirsing, G., Luz, A.: Hydrogeologischer Bau und Aquifereigenschaften der Lockergesteine im Oberrheingraben (Baden-Württemberg). LGRB, Freiburg i. Brsg. (2007)

Zollinger, G., Mäckel, R.: Quartäre Geomorphodynamik im Einzugsgebiet des Sulzbaches und der Möhlin, Südbaden. Ber. Naturf. Ges. Freiburg i. Brsg.., S. 81-98 (1989)

Hinweis des Verlags Der Verlag bleibt in Hinblick auf geografische Zuordnungen und Gebietsbezeichnungen in veröffentlichten Karten und Institutsadressen neutral. 